\title{
Forbidden Configurations: Exact bounds determined by critical substructures
}

\author{
R. P. Anstee* and S. N. Karp ${ }^{\dagger}$ \\ Mathematics Department \\ The University of British Columbia \\ Vancouver, B.C. Canada V6T 1Z2 \\ anstee@math.ubc.ca skarp@uwaterloo.ca
}

Submitted: Aug 5, 2009; Accepted: Mar 18, 2010; Published: Mar 29, 2010

Mathematics Subject Classification: 05D05

\begin{abstract}
We consider the following extremal set theory problem. Define a matrix to be simple if it is a $(0,1)$-matrix with no repeated columns. An $m$-rowed simple matrix corresponds to a family of subsets of $\{1,2, \ldots, m\}$. Let $m$ be a given integer and $F$ be a given (0,1)-matrix (not necessarily simple). We say a matrix $A$ has $F$ as a configuration if a submatrix of $A$ is a row and column permutation of $F$. We define forb $(m, F)$ as the maximum number of columns that a simple $m$-rowed matrix $A$ can have subject to the condition that $A$ has no configuration $F$. We compute exact values for forb $(m, F)$ for some choices of $F$ and in doing so handle all $3 \times 3$ and some $k \times 2(0,1)$-matrices $F$. Often forb $(m, F)$ is determined by forb $\left(m, F^{\prime}\right)$ for some configuration $F^{\prime}$ contained in $F$ and in that situation, with $F^{\prime}$ being minimal, we call $F^{\prime}$ a critical substructure.
\end{abstract}

Keywords: VC-dimension, (0,1)-matrices, forbidden configurations, trace

\section{Introduction}

We define a simple matrix as a $(0,1)$-matrix with no repeated columns. Assume we are given a $k \times \ell(0,1)$-matrix $F$. We say that a matrix $A$ has $F$ as a configuration if $A$ has

${ }^{*}$ Research supported in part by NSERC. The first author gratefully thanks the Mathematics Department of the University of South Carolina, Columbia, SC, USA for hosting me during my sabbatical in 2008 when this work was initiated.

${ }^{\dagger}$ Undergraduate at $\mathrm{U}$ of Waterloo with research at UBC supported by NSERC USRA and NSERC of first author 
a $k \times \ell$ submatrix which is a row and column permutation of $F$ and so $F$ is referred to as a configuration in $A$ (sometimes called trace). Many $F$ considered in this paper are non-simple.

For a matrix $A$, we define $|A|$ to denote the number of columns in $A$. We define forb $(m, F)$ as the smallest value (depending on $m$ and $F$ ) so that if $A$ is a simple $m$-rowed matrix and $A$ has no configuration $F$ then $|A| \leqslant \operatorname{forb}(m, F)$. Alternatively forb $(m, F)$ is the smallest value so that if $A$ is an $m \times(\operatorname{forb}(m, F)+1)$ simple matrix then $A$ must have $F$ as a configuration. Exact bounds require greater care and deeper understanding to achieve than asymptotic results although it is also true that asymptotic results provide broad understanding. Theorem 9.1 gives a forbidden configuration for which an exact bound would be troublesome. We hope that the results given here may provoke further study. An interested reader might look at the survey of results in [1]. We obtain a wealth of exact bounds. One consequence of our results are exact bounds for all $3 \times 3$ forbidden configurations given in Table 1 and Table 2.

Table 1. Simple $3 \times 3 F$

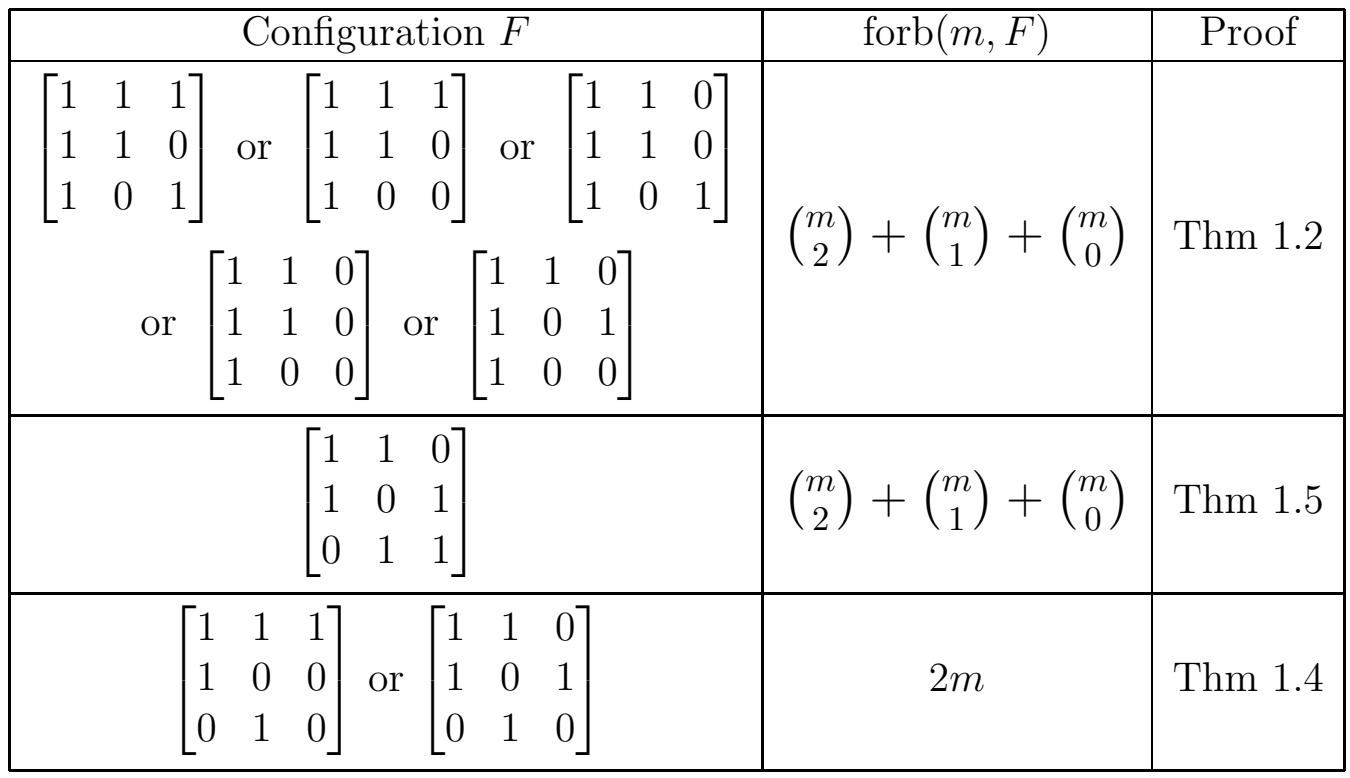

The reader may check that all $3 \times 3$ configurations or their $(0,1)$-complements have been included in the two tables. We have enumerated the matrices based on columns of sum 3 first and on columns of sum 2 second. Note that if $F^{c}$ is the $(0,1)$-complement of $F$, then forb $(m, F)=\operatorname{forb}\left(m, F^{c}\right)$. We note that there may be other attractive ways to list the matrices such as representing the 5 configurations in the first line of the table by a single object any possible completion of which is one of the 5 configurations

$$
\left[\begin{array}{lll}
1 & 1 & 0 \\
1 & 0 & \\
1 & &
\end{array}\right] .
$$

We hope the table of results makes the problem of forbidden configurations more accessible while indicating how general bounds prove many exact results. In each case, 
there may be a short argument to see how to apply the Theorem.

In the following table note that we also define the configurations $F_{1}, F_{2}, F_{3}, F_{4}, F_{5}$.

Table 2. Non-simple $3 \times 3 F$

\begin{tabular}{|c|c|c|c|c|c|c|}
\hline \multicolumn{5}{|c|}{ Configuration } & forb $(m, F)$ & \multirow{2}{*}{\begin{tabular}{|c} 
Proof \\
Thm 1.6
\end{tabular}} \\
\hline & & & {$\left[\begin{array}{lll}1 & 1 & 1 \\
1 & 1 & 1 \\
1 & 1 & 1\end{array}\right]$} & & $\frac{5}{4}\left(\begin{array}{c}m \\
3\end{array}\right)+\left(\begin{array}{c}m \\
2\end{array}\right)+\left(\begin{array}{c}m \\
1\end{array}\right)+\left(\begin{array}{c}m \\
0\end{array}\right)$ & \\
\hline$\left[\begin{array}{ll}1 & 1 \\
1 & 1 \\
1 & 1\end{array}\right]$ & $\left.\begin{array}{l}1 \\
1 \\
0\end{array}\right]$ & , & $\left.\begin{array}{lll}1 & 1 & 1 \\
1 & 1 & 0 \\
1 & 1 & 0\end{array}\right]$ or & {$\left[\begin{array}{lll}1 & 1 & 0 \\
1 & 1 & 0 \\
1 & 1 & 0\end{array}\right]$} & $\left(\begin{array}{c}m \\
3\end{array}\right)+\left(\begin{array}{c}m \\
2\end{array}\right)+\left(\begin{array}{c}m \\
1\end{array}\right)+\left(\begin{array}{c}m \\
0\end{array}\right)$ & Thm 1.3 \\
\hline & & & {$\left[\begin{array}{lll}1 & 1 & 1 \\
1 & 1 & 1 \\
0 & 0 & 0\end{array}\right]$} & & $\frac{4}{3}\left(\begin{array}{c}m \\
2\end{array}\right)+\left(\begin{array}{c}m \\
1\end{array}\right)+\left(\begin{array}{c}m \\
0\end{array}\right)+\left(\begin{array}{c}m \\
m\end{array}\right)$ & Thm 1.7 \\
\hline & & $F_{1}=$ & $=\left[\begin{array}{lll}1 & 1 & 1 \\
1 & 1 & 1 \\
1 & 0 & 0\end{array}\right]$ & & $\frac{4}{3}\left(\begin{array}{c}m \\
2\end{array}\right)+\left(\begin{array}{c}m \\
1\end{array}\right)+\left(\begin{array}{c}m \\
0\end{array}\right)$ & Thm 8.1 \\
\hline $\begin{array}{c}F_{2}= \\
F_{4}=\end{array}$ & $\begin{array}{l}{\left[\begin{array}{l}1 \\
1 \\
1\end{array}\right.} \\
{\left[\begin{array}{l}1 \\
1 \\
0\end{array}\right.}\end{array}$ & $\begin{array}{ll}1 & 1 \\
0 & 0 \\
0 & 0 \\
1 & 1 \\
1 & 0 \\
0 & 1\end{array}$ & $\left.\begin{array}{l}], F_{3}=[ \\
1 \\
0 \\
0\end{array}\right]$ or $F_{5}=$ & 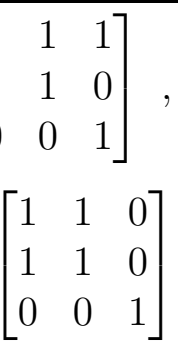 & $\left(\begin{array}{c}m \\
2\end{array}\right)+\left(\begin{array}{c}m \\
1\end{array}\right)+\left(\begin{array}{c}m \\
0\end{array}\right)+\left(\begin{array}{c}m \\
m\end{array}\right)$ & Thm 3.2 \\
\hline
\end{tabular}

If $F^{\prime}$ is a configuration in $F$ then we note that any matrix $A$ with no $F^{\prime}$ has no $F$ and so forb $\left(m, F^{\prime}\right) \leqslant \operatorname{forb}(m, F)$. We define a critical substructure for a forbidden configuration $F$ to be a minimal $F^{\prime}$ that is a configuration in $F$ and so that forb $\left(m, F^{\prime}\right)=\operatorname{forb}(m, F)$. One application is to note that if $F^{\prime \prime}$ is a configuration of $F$ and $F^{\prime}$ is a configuration of $F^{\prime \prime}$ then we obtain a 'sandwich' result that forb $\left(m, F^{\prime}\right)=\operatorname{forb}\left(m, F^{\prime \prime}\right)=\operatorname{forb}(m, F)$. This is applied to Theorem 1.4 to obtain two bounds in Table 1 . Note that the $4 \times 4$ $F_{10}$ in Theorem 3.4 has a $3 \times 2$ critical substructure and so yields several other exact bounds. Another application is when faced with a given $F$, it may be helpful to consider configurations contained in $F$ and, among those with the largest bound, attempt to prove one is a critical substructure. This was done in Theorem 8.1. We do not explore the related idea which would consider a given $F^{\prime}$ and seek maximal $F^{\prime}$ 's for which $F^{\prime}$ is a critical substructure. For some pairs $F^{\prime}, F$, the largest $m$-rowed matrix with no $F$ also has no $F^{\prime}$. The paper considers some basic choices for $F^{\prime}$ and obtains some $F$ with forb $\left(m, F^{\prime}\right)=\operatorname{forb}(m, F)$. We think this concept of critical substructures deserves to be highlighted as one approach in analyzing forbidden configurations.

It is helpful to define $\mathbf{1}_{k} \mathbf{0}_{\ell}$ as the $(k+\ell) \times 1$ column of $k 1$ 's on top of $\ell 0$ 's. In addition 
let $\mathbf{1}_{k}=\mathbf{1}_{k} \mathbf{0}_{0}$ and $\mathbf{0}_{k}=\mathbf{1}_{0} \mathbf{0}_{k}$. For a positive integer $q$ and a matrix $F$, define $q \cdot F$ as the concatenation of $q$ copies of $F$ so that $q \cdot \mathbf{1}_{k} \mathbf{0}_{\ell}$ is the $q \times(k+\ell)$ matrix of $q$ copies of $\mathbf{1}_{k} \mathbf{0}_{\ell}$. In many of the examples above we can find non-trivial critical substructures. In Table 1, we see that the first 5 configurations all have a critical substructure $\mathbf{1}_{3}$. In Table 2 we see that the second to fourth configurations share a critical substructure $2 \cdot \mathbf{1}_{3}$ and the sixth has a critical substructure $3 \cdot \mathbf{1}_{2}$. We also note that $F_{2}$ has $2 \cdot \mathbf{1}_{1} \mathbf{0}_{2}$ as a critical substructure and $F_{3}, F_{4}, F_{5}$ have critical substructure $2 \cdot \mathbf{1}_{2} \mathbf{0}_{1}$. Define

$\operatorname{ext}(m, F)=\{A: A$ is a $m \times \operatorname{forb}(m, F)$ simple matrix with no configuration $F\}$.

It is often the case that for a unique critical substructure $F^{\prime}$ of $F$, that $\operatorname{ext}(m, F)=$ $\operatorname{ext}\left(m, F^{\prime}\right)$ or if $F$ has two critical substructures $F^{\prime}, F^{\prime \prime}$ then $\operatorname{ext}(m, F)=\operatorname{ext}\left(m, F^{\prime}\right) \cup$ $\operatorname{ext}\left(m, F^{\prime \prime}\right)$. The following result presented without proof will be used extensively in the paper.

Theorem 1.1 Let $p, q$ be nonnegative integers with $p \geqslant q$. Assume $m \geqslant p+q$. Then $\operatorname{forb}\left(m, \mathbf{1}_{p} \mathbf{0}_{q}\right)=\sum_{i=0}^{p-1}\left(\begin{array}{c}m \\ i\end{array}\right)+\sum_{i=0}^{q-1}\left(\begin{array}{c}m \\ i\end{array}\right)$ and $\operatorname{ext}\left(m, \mathbf{1}_{p} \mathbf{0}_{q}\right)$ consists of the matrices which have all columns of at most $p-1$ 1's and all columns with at most $q-10$ 's. If we assume $m<p+q$, then forb $\left(m, \mathbf{1}_{p} \mathbf{0}_{q}\right)=2^{m}$.

It is convenient to use the language of matrix theory and sets. Let $[m]=\{1,2, \ldots, m\}$. An $m \times n$ simple matrix $A$ can be thought of a family $\mathcal{A}$ of $n$ subsets $S_{1}, S_{2}, \ldots, S_{n}$ of $[m]=\{1,2, \ldots, m\}$ where $i \in S_{j}$ if and only if the $(i, j)$ entry of $A$ is 1 . For a set $S \subseteq[m]$ and an integer $k$, we define

$$
2^{S}=\{B \subseteq[m]: B \subseteq S\}, \quad\left(\begin{array}{l}
S \\
k
\end{array}\right)=\{B \subseteq S:|B|=k\}
$$

Moreover define $K_{k}$ to be the $k \times 2^{k}$ simple matrix corresponding to $2^{[k]}$ and define $K_{k}^{l}$ to be the $k \times\left(\begin{array}{l}k \\ l\end{array}\right)$ simple matrix corresponding to $\left(\begin{array}{c}{[k]} \\ l\end{array}\right)$. Given two $m$-rowed matrices $A, B$, we use the notation $[A B]$ (this is not $A$ times $B$ !) to denote the matrix obtained by concatenating the two matrices. This would be the analogue of set union of our families. For example we have $\operatorname{ext}\left(m, \mathbf{1}_{k}\right)$ corresponding to the family $\left(\begin{array}{c}{[m]} \\ 0\end{array}\right) \cup\left(\begin{array}{c}{[m]} \\ 1\end{array}\right) \cup \cdots \cup\left(\begin{array}{c}{[m]} \\ k-1\end{array}\right)$ and to the matrix $\left[K_{m}^{0} K_{m}^{1} K_{m}^{2} \cdots K_{m}^{k-1}\right]$.

Most $3 \times 3$ forbidden configurations can be handled by the general theorems given below specialized to 3 rows.

Theorem 1.2 Sauer[10], Perles and Shelah[11], Vapnik and Chervonenkis[12]. Let $k$ be given.

$$
\operatorname{forb}\left(m, \mathbf{1}_{k}\right)=\operatorname{forb}\left(m, K_{k}\right)=\left(\begin{array}{c}
m \\
k-1
\end{array}\right)+\left(\begin{array}{c}
m \\
k-2
\end{array}\right)+\cdots+\left(\begin{array}{c}
m \\
0
\end{array}\right) .
$$

Theorem 1.3 Gronau [8]. Let $k$ be given.

$$
f o r b\left(m, 2 \cdot \mathbf{1}_{k}\right)=\operatorname{forb}\left(m, 2 \cdot K_{k}\right)=\left(\begin{array}{c}
m \\
k
\end{array}\right)+\left(\begin{array}{c}
m \\
k-1
\end{array}\right)+\left(\begin{array}{c}
m \\
k-2
\end{array}\right)+\cdots+\left(\begin{array}{c}
m \\
0
\end{array}\right) .
$$


Theorem 1.4 Anstee, Griggs, Sali[4].

$$
\operatorname{forb}\left(m,\left[\begin{array}{ll}
1 & 1 \\
0 & 1 \\
1 & 0
\end{array}\right]\right)=\operatorname{forb}\left(m,\left[\begin{array}{llll}
1 & 1 & 1 & 0 \\
0 & 1 & 0 & 1 \\
1 & 0 & 0 & 0
\end{array}\right]\right)=2 m .
$$

Theorem 1.5 Ryser[9].

$$
\operatorname{forb}\left(m,\left[\begin{array}{ccc}
1 & 1 & 0 \\
1 & 0 & 1 \\
0 & 1 & 1
\end{array}\right]\right)=\left(\begin{array}{c}
m \\
2
\end{array}\right)+\left(\begin{array}{c}
m \\
1
\end{array}\right)+\left(\begin{array}{c}
m \\
0
\end{array}\right) .
$$

Theorem 1.6 Anstee, Füredi[5]. Let $t, k$ be given with $t \geqslant 2$.

$$
\operatorname{forb}\left(m, t \cdot \mathbf{1}_{k}\right)=\operatorname{forb}\left(m, t \cdot K_{k}\right) \leqslant \frac{t+k-1}{k+1}\left(\begin{array}{c}
m \\
k
\end{array}\right)+\left(\begin{array}{c}
m \\
k-1
\end{array}\right)+\cdots+\left(\begin{array}{c}
m \\
0
\end{array}\right),
$$

with equality if there exists a simple $k$-design on $m$ points with block size $k+1$ and $\lambda=t-2$.

Theorem 1.7 Anstee, Barekat[2]. Let $m, k, t$ be given with $m \equiv 1,3(\bmod 6)$. Then there exists an integer $M$ so that for $m \geqslant M$,

$$
\operatorname{forb}\left(m, t \cdot \mathbf{1}_{2} \mathbf{0}_{1}\right)=\frac{t+1}{3}\left(\begin{array}{c}
m \\
2
\end{array}\right)+\left(\begin{array}{c}
m \\
1
\end{array}\right)+\left(\begin{array}{c}
m \\
0
\end{array}\right)+\left(\begin{array}{c}
m \\
m
\end{array}\right) .
$$

A helpful notation for two columned forbidden configurations is to define $F_{a, b, c, d}$ to be the $(a+b+c+d) \times 2$ matrix consisting of $a$ rows [11], $b$ rows [10], $c$ rows [01] and $d$ rows [00]. One can restrict attention to cases with $a \geqslant d$ and $b \geqslant c$. An example is $2 \cdot \mathbf{1}_{p} \mathbf{0}_{q}=F_{p, 0,0, q}$. Complete asymptotic results for forb $\left(m, F_{a, b, c, d}\right)$ are in [7]. Given the difficulty and nature of the proofs in [7] we do not expect to obtain exact bounds for all choices $a, b, c, d$. Theorem 9.1 indicates that $F_{2,1,1,0}$ is a hard case. Exact bounds for other $4 \times 2 F$ are in [3].

We are able to prove exact bounds for certain $F_{a, b, c, d}$. We note that $F_{a, b, c, d}$ has configurations $\mathbf{1}_{a+b} \mathbf{0}_{c+d}$ and $\mathbf{1}_{a+c} \mathbf{0}_{b+d}$ (corresponding to the two columns). Sometimes one or the other or both are critical substructures. The first result is an easy pigeonhole argument from Theorem 1.3 in [3]. We observe that a simple matrix has a configuration $F_{p, 0,0, q}$ if and only if it has $F_{p, 1,0, q}$.

Theorem 1.8 Let $p, q$ be given with $p \geqslant q$. Then

$$
\left.\operatorname{forb}\left(m, 2 \cdot \mathbf{1}_{p} \mathbf{0}_{q}\right)\right)=\operatorname{forb}\left(m, F_{p, 0,0, q}\right)=\operatorname{forb}\left(m, F_{p, 1,0, q}\right)=\sum_{i=0}^{p}\left(\begin{array}{c}
m \\
i
\end{array}\right)+\sum_{i=m-q+1}^{m}\left(\begin{array}{c}
m \\
i
\end{array}\right),
$$

and moreover for $p>q$,

$$
\operatorname{ext}\left(m, 2 \cdot \mathbf{1}_{p} \mathbf{0}_{q}\right)=\operatorname{ext}\left(m, F_{p, 0,0, q}\right)=\operatorname{ext}\left(m, F_{p, 1,0, q}\right)=\operatorname{ext}\left(m, \mathbf{1}_{p+1} \mathbf{0}_{q}\right) .
$$


Proof: The result on $\operatorname{ext}\left(m, 2 \cdot \mathbf{1}_{p} \mathbf{0}_{q}\right)$ follows easily from the pigeonhole proof of forb $(m, 2$. $\left.\mathbf{1}_{p} \mathbf{0}_{q}\right)$ in [3]. For $p=q$, the extremal matrices can be more varied.

Theorem $1.9[6]$. We have forb $\left(m, F_{1,1,1,1}\right)=4 m-4$.

The following summarizes our new exact bounds handling many cases with $c, d \in\{0,1\}$. We note that for $b \geqslant 1$, forb $\left(m, F_{a, b, 0,0}\right)=\operatorname{forb}\left(m, K_{a+b}\right)$ and so we can use Theorem 1.2.

Theorem 1.10 Let $m, a, b$ be given integers. For $m \geqslant 1$,

$$
\begin{gathered}
\operatorname{forb}\left(m, F_{a, b, 1,0}\right)=\operatorname{forb}\left(m, \mathbf{1}_{a+b} \mathbf{0}_{1}\right) \text { for } a \geqslant 1, b \geqslant 2 \text { or } a=0, b \geqslant 3, \\
\operatorname{forb}\left(m, F_{a, b, 0,1}\right)=\operatorname{forb}\left(m, \mathbf{1}_{a+b} \mathbf{0}_{1}\right) \text { for } a \geqslant 1, b \geqslant 1, \\
\operatorname{forb}\left(m, F_{a, b, 1,1}\right)=\operatorname{forb}\left(m, \mathbf{1}_{a+b} \mathbf{0}_{2}\right) \text { for } a \geqslant 1, b \geqslant 2 .
\end{gathered}
$$

Proof: It was already shown in [3] that for $b \geqslant 3$, forb $\left(m, F_{0, b, 1,0}\right)=\operatorname{forb}\left(m, \mathbf{1}_{b} \mathbf{0}_{1}\right)$. Theorem 5.6 handles forb $\left(m, F_{a, b, 1,0}\right)$ for $a \geqslant 1, b \geqslant 2$. Theorem 5.6 handles forb $\left(m, F_{a, b, 0,1}\right)$ for $a \geqslant 2, b \geqslant 1$, Theorem 1.8 handles forb $\left(m, F_{1,1,0,1}\right)$ and Theorem 6.5 handles forb $(m$, $\left.F_{1, b, 0,1}\right)$ for $b \geqslant 2$. Theorem 7.2 handles forb $\left(m, F_{a, b, 1,1}\right)$ for $a \geqslant 1, b \geqslant 2$.

We often compute $\operatorname{ext}\left(m, F_{a, b, c, d}\right)$ as part of the proof. We have organized the paper by critical substructures. Section 3 considers critical substructures $2 \cdot \mathbf{1}_{k-1} \mathbf{0}_{1}$ and handles configurations $F_{2}, F_{3}, F_{4}, F_{5}$ among others. Section 4 considers critical substructures $\mathbf{1}_{k}$. Section 5 and Section 6 considers critical substructures $\mathbf{1}_{k-1} \mathbf{0}_{1}$. Section 7 considers critical substructures $\mathbf{1}_{k-2} \mathbf{0}_{2}$. The proof techniques are mostly clever inductions, the most basic of which are in Section 2. Section 8 considers critical substructures $3 \cdot \mathbf{1}_{k-1}$ (and handles $F_{1}$ when $k=3$ ) using an interesting matching argument. We list a number of open problems: Problem 5.1, Problem 10.1, Problem 10.2, Problem 10.3.

\section{Induction Arguments}

Many of our arguments use induction. If $A$ is a simple $m$-rowed matrix, then when we delete row 1 from $A$, the resulting matrix need not be simple and may have repeated columns. We decompose $A$, after permuting its columns, as

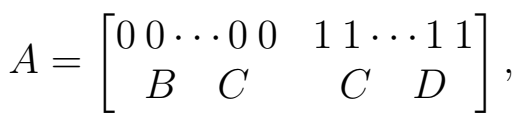

where $C$ is the matrix of columns which are repeated when the first row of $A$ is deleted. We call this the standard decomposition of $A$. Note that above we have decomposed $A$ based on its first row. Sometimes we will consider decompositions of $A$ based on another row. We thus define the row-r decomposition of $A$ to be the decomposition (1) performed after row $r$ and row 1 are switched. We denote the three resulting matrices by $B_{r}, C_{r}$, and $D_{r}$. That is,

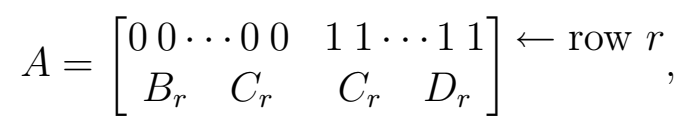


where $C_{r}$ is the matrix of columns which are repeated when row $r$ is deleted from $A$.

If $A$ avoids a configuration $F$, then we can make two observations about the matrices $B_{r}, C_{r}$, and $D_{r}$. First, $\left[B_{r} C_{r} D_{r}\right]$ is a simple $(m-1)$-rowed matrix with no $F$. Second, $C_{r}$ is a simple $(m-1)$-rowed matrix which avoids many submatrices of $F$. For example, if

$$
F=\left[\begin{array}{lll}
1 & 0 & 0 \\
0 & 1 & 0 \\
0 & 0 & 0
\end{array}\right]
$$

then by stripping off the second row from $F$, we see that $C_{r}$ has no $\left[\begin{array}{ll}1 & 0 \\ 0 & 0\end{array}\right]$ or else $A$ has $F$. More formally we can describe this computation as follows.

Lemma 2.1 Let $k$ be given and let $F$ be a $k$-rowed matrix. For each $s \in[k]$, decompose F using (2) as

$$
F=\left[\begin{array}{cccc}
00 \cdots 0 & 00 \cdots 0 & 11 \cdots 1 & 11 \cdots 1 \\
B_{s}(F) & C_{s}(F) & C_{s}(F) & D_{s}(F)
\end{array}\right] \leftarrow \text { row } s
$$

Then if $A$ is a simple matrix with no configuration $F$, then in the row decomposition of $A$ of (1), we deduce that $C$ has no configurations $\left[B_{s}(F) C_{s}(F) D_{s}(F)\right]$ for each $s \in[k]$.

The following basic induction facts come from Pascal's identity and the observation that $|A|=\left|\left[B_{r} C_{r} D_{r}\right]\right|+\left|C_{r}\right|$ in $(2)$.

Remark 2.2 Assume $p \geqslant 1$. Then forb $\left(m, \mathbf{1}_{p}\right)=\operatorname{forb}\left(m-1, \mathbf{1}_{p}\right)+\operatorname{forb}\left(m-1, \mathbf{1}_{p-1}\right)$. If we also assume $q \geqslant 1$, then forb $\left(m, \mathbf{1}_{p} \mathbf{0}_{q}\right)=\operatorname{forb}\left(m-1, \mathbf{1}_{p} \mathbf{0}_{q}\right)+\operatorname{forb}\left(m-1, \mathbf{1}_{p-1} \mathbf{0}_{q-1}\right)$. Let $p, q$ be given non-negative integers $p \geqslant q$ with $p \geqslant 1, q \geqslant 0$. Assume $A$ is an $m$-rowed simple matrix. Let $r$ be chosen with $r \in[m]$ and consider the row $r$ decomposition (2) of A. Assume

$$
\left|\left[B_{r} C_{r} D_{r}\right]\right| \leqslant \operatorname{forb}\left(m-1, \mathbf{1}_{p} \mathbf{0}_{q}\right) .
$$

Assume that either for $q=0$,

$$
\left|C_{r}\right| \leqslant \operatorname{forb}\left(m-1, \mathbf{1}_{p-1}\right)
$$

or for $q \geqslant 1$,

$$
\left|C_{r}\right| \leqslant \operatorname{forb}\left(m-1, \mathbf{1}_{p-1} \mathbf{0}_{q-1}\right) .
$$

Then

$$
|A| \leqslant \operatorname{forb}\left(m, \mathbf{1}_{p} \mathbf{0}_{q}\right) .
$$




\section{Critical substructure $2 \cdot \mathbf{1}_{k-1} \mathbf{0}_{1}$}

In this section we find some $k$-rowed $F$ which contain $2 \cdot \mathbf{1}_{k-1} \mathbf{0}_{1}$ for which $\operatorname{ext}(m, F)=$ $\operatorname{ext}\left(m, 2 \cdot \mathbf{1}_{k-1} \mathbf{0}_{1}\right)$. Given that $F$ has a repeated column, the results are independent of Theorem 1.2. Note that Theorem 1.8 handles $F=2 \cdot \mathbf{1}_{k-1} \mathbf{0}_{1}$. We outline a general induction argument for configurations which contain $2 \cdot \mathbf{1}_{k-1} \mathbf{0}_{1}$, and then apply it to get the bounds and extremal matrices for some cases, settling the remaining entries in Table 2.

Lemma 3.1 Suppose that $k \geqslant 2$, and $G$ is a $k$-rowed simple matrix which does not have the column $\mathbf{1}_{k-1} \mathbf{0}_{1}$ but may have other columns of column sum $k-1$. Let $F$ be the $k$-rowed matrix

$$
F=\left[2 \cdot \mathbf{1}_{k-1} \mathbf{0}_{1} \mid G\right]=k-1\left\{\left[\begin{array}{ccc}
1 & 1 & \\
\vdots & \vdots & \\
1 & 1 & \\
0 & 0 &
\end{array}\right]\right.
$$

and let $m \geqslant k+1$. Suppose that we know the following two things for all $A \in \operatorname{ext}(m, F)$ : (i) $\operatorname{ext}(m-1, F)=\operatorname{ext}\left(m-1,2 \cdot \mathbf{1}_{k-1} \mathbf{0}_{1}\right)=\operatorname{ext}\left(m-1, \mathbf{1}_{k} \mathbf{0}_{1}\right)$.

(ii) For every $r \in[m]$ with $\left|C_{r}\right|>$ forb $\left(m-1, \mathbf{1}_{k-1}\right)$ in the row-r decomposition (2) of $A$ we have $C_{r} \in \operatorname{ext}\left(m-1, \mathbf{1}_{k-1} \mathbf{0}_{1}\right)$.

Then we may conclude

$$
\operatorname{ext}(m, F)=\operatorname{ext}\left(m, 2 \cdot \mathbf{1}_{k-1} \mathbf{0}_{1}\right)=\operatorname{ext}\left(m, \mathbf{1}_{k} \mathbf{0}_{1}\right)
$$

Proof: Assume $A \in \operatorname{ext}(m, F)$. First we show that $\left[B_{r} C_{r} D_{r}\right] \in \operatorname{ext}\left(m-1, \mathbf{1}_{k} \mathbf{0}_{1}\right)$ in the row- $r$ decomposition of $A$ for all $r \in[m]$. Note that by our assumption (i), it will suffice to show that $\left|\left[B_{r} C_{r} D_{r}\right]\right| \geqslant \operatorname{forb}\left(m-1, \mathbf{1}_{k} \mathbf{0}_{1}\right)=\operatorname{forb}(m-1, F)$ and note that $\left[B_{r} C_{r} D_{r}\right]$ has no $F$.

We proceed by contradiction and suppose for some $r \in[m]$ that in the row- $r$ decomposition of $A$ that $\left|\left[B_{r} C_{r} D_{r}\right]\right|<\operatorname{forb}\left(m-1, \mathbf{1}_{k} \mathbf{0}_{1}\right)$. Since $F$ contains $2 \cdot \mathbf{1}_{k-1} \mathbf{0}_{1}$, we have $|A|=\operatorname{forb}(m, F) \geqslant \operatorname{forb}\left(m, 2 \cdot \mathbf{1}_{k-1} \mathbf{0}_{1}\right)=\operatorname{forb}\left(m, \mathbf{1}_{k} \mathbf{0}_{1}\right)$. Hence $\left|C_{r}\right|=|A|-\left|\left[B_{r} C_{r} D_{r}\right]\right|>$ forb $\left(m, \mathbf{1}_{k} \mathbf{0}_{1}\right)-\operatorname{forb}\left(m-1, \mathbf{1}_{k} \mathbf{0}_{1}\right)=\operatorname{forb}\left(m, \mathbf{1}_{k-1}\right)$ using Remark $2.2(p=k, q=1)$. Then by our assumption (ii), $C_{r} \in \operatorname{ext}\left(m-1, \mathbf{1}_{k-1} \mathbf{0}_{1}\right)$, and hence using the column of 1 's and the columns of column sum at most $k-2$, we deduce $C_{r}$ has the configuration $K_{k-1}$ on each $(k-1)$-set of its rows.

Now note that we can write $F$ as

$$
F=k-1\left\{\left[\begin{array}{c|c|c}
1 & & \\
\vdots & G_{1} & G_{2} \\
1 & & \\
1 & & \\
0 & 00 \cdots 0 & 11 \cdots 1
\end{array}\right]=k-1\left\{\left[\begin{array}{c|c|c}
1 & 11 \cdots 1 & 00 \cdots 0 \\
1 & & \\
\vdots & G_{3} & G_{4} \\
1 & &
\end{array}\right],\right.\right.
$$

where $G_{1}, G_{2}, G_{3}, G_{4}$ are each $k-1$-rowed simple matrices. Thus $C_{r}$ has every row permutation of $G_{1}, G_{2}, G_{3}$ and $G_{4}$ on each $(k-1)$-tuple of its rows. Now if we look for a copy 
of $F$ where the bottom row of the first matrix above is in row $r$ of $A$, we deduce that $B_{r}$ has no $\mathbf{1}_{k-1}$ else $A$ has $F$. Similarly, if we look for the top row of the second matrix above in row $r$ of $A$, we see that $D_{r}$ has no $\mathbf{1}_{k-2} \mathbf{0}_{1}$. But $C_{r} \in \operatorname{ext}\left(m-1, \mathbf{1}_{k-1} \mathbf{0}_{1}\right)$ and so $C_{r}$ has all columns which avoid $\mathbf{1}_{k-1} \mathbf{0}_{1}$ and hence all columns which avoid $\mathbf{1}_{k-1}$ or $\mathbf{1}_{k-2} \mathbf{0}_{1}$. Since $B_{r}$ and $D_{r}$ share no columns with $C_{r}$, we conclude that $B_{r}$ and $D_{r}$ are empty. Hence

$$
|A|=2\left|C_{r}\right|=2 \cdot \operatorname{forb}\left(m-1, \mathbf{1}_{k-1} \mathbf{0}_{1}\right)=2 \sum_{i=0}^{k-2}\left(\begin{array}{c}
m-1 \\
i
\end{array}\right)+2=\sum_{i=0}^{k-2}\left(\begin{array}{c}
m \\
i
\end{array}\right)+\left(\begin{array}{c}
m-1 \\
k-2
\end{array}\right)+2 .
$$

Now $|A| \geqslant \operatorname{forb}\left(m, 2 \cdot \mathbf{1}_{k-1} \mathbf{0}_{1}\right)=\sum_{i=0}^{k-1}\left(\begin{array}{c}m \\ i\end{array}\right)+1$, whence

$$
\left(\begin{array}{c}
m-1 \\
k-2
\end{array}\right) \geqslant\left(\begin{array}{c}
m \\
k-1
\end{array}\right)-1=\left(\begin{array}{c}
m-1 \\
k-2
\end{array}\right)+\left(\begin{array}{c}
m-1 \\
k-1
\end{array}\right)-1>\left(\begin{array}{c}
m-1 \\
k-2
\end{array}\right),
$$

a contradiction.

We now conclude $\left[B_{r} C_{r} D_{r}\right] \in \operatorname{ext}\left(m-1, \mathbf{1}_{k} \mathbf{0}_{1}\right)$. For all $r \in[m]$ it follows that $\left[B_{r} C_{r} D_{r}\right]$ has the configuration $K_{k}$ on each $k$-tuple of its rows. Since this is true for all $r \in[m]$, we conclude that $A$ has $K_{k}$ on each $k$-tuple of rows, and hence $A$ has every row permutation of $G$, on each $k$-tuple of its rows. Thus $A$ avoids $2 \cdot \mathbf{1}_{k-1} \mathbf{0}_{1}$, the first two columns of $F$. The result now follows from Theorem 1.8.

We apply Lemma 3.1 to establish the extremal matrices for three specific 3-rowed Forbidden Configurations.

Theorem 3.2 Let

$$
F_{6}=\left[\begin{array}{lllll}
1 & 1 & 1 & 0 & 0 \\
1 & 1 & 0 & 1 & 0 \\
0 & 0 & 1 & 0 & 0
\end{array}\right], F_{7}=\left[\begin{array}{lllll}
1 & 1 & 1 & 0 & 0 \\
1 & 1 & 0 & 1 & 0 \\
0 & 0 & 0 & 0 & 0
\end{array}\right], F_{8}=\left[\begin{array}{llllll}
1 & 1 & 1 & 1 & 0 & 0 \\
1 & 1 & 0 & 0 & 0 & 0 \\
0 & 0 & 1 & 0 & 1 & 0
\end{array}\right] .
$$

Then for $m \geqslant 3$,

$$
\operatorname{ext}\left(m, F_{6}\right)=\operatorname{ext}\left(m, F_{7}\right)=\operatorname{ext}\left(m, F_{8}\right)=\operatorname{ext}\left(m, 2 \cdot \mathbf{1}_{2} \mathbf{0}_{1}\right)=\operatorname{ext}\left(m, \mathbf{1}_{3} \mathbf{0}_{1}\right) .
$$

Proof: We induct on $m$, using Lemma 3.1 with $k=3$. Our base case $m=3$ is clear, since any simple 3 -rowed matrix avoids $F_{6}, F_{7}$ and $F_{8}$.

Now suppose that the result is true for $m-1$, where $m \geqslant 4$. Let $A$ be matrix in $\operatorname{ext}\left(m, F_{6}\right) \cup \operatorname{ext}\left(m, F_{7}\right) \cup \operatorname{ext}\left(m, F_{8}\right)$. Configurations $F_{6}, F_{7}$ and $F_{8}$ are of the form given in the statement of Lemma 3.1 with $k=3$, so it will suffice to show that the two hypotheses of the Lemma hold. The statement (i) follows from our induction hypothesis. We now show that (ii) holds. That is, we suppose $r \in[m]$ such that, in the row- $r$ decomposition (2) of $A,\left|C_{r}\right|>\operatorname{forb}\left(m-1, \mathbf{1}_{2}\right)=m$, and we show that then $C_{r} \in \operatorname{ext}\left(m-1, \mathbf{1}_{2} \mathbf{0}_{1}\right)$.

We may use Lemma 2.1 for the configurations $F_{6}, F_{7}$ or $F_{8}$ to verify that regardless of which configuration $F_{6}, F_{7}, F_{8}$ is forbidden in $A$, then $C_{r}$ has neither

$$
L_{1}=\left[\begin{array}{llll}
1 & 1 & 0 & 0 \\
0 & 0 & 1 & 0
\end{array}\right] \quad \text { nor } \quad L_{2}=\left[\begin{array}{lllll}
1 & 1 & 1 & 1 & 0 \\
1 & 1 & 0 & 0 & 0
\end{array}\right] \quad \text { nor } \quad L_{3}=\left[\begin{array}{lllll}
1 & 1 & 1 & 0 & 0 \\
1 & 1 & 0 & 1 & 0
\end{array}\right]
$$


By Theorem 1.2 we have forb $\left(m-1, \mathbf{1}_{2}\right)=\operatorname{forb}\left(m-1, K_{2}\right)$, so $C_{r}$ contains a copy of $K_{2}=\left[\begin{array}{llll}1 & 1 & 0 & 0 \\ 1 & 0 & 1 & 0\end{array}\right]$ without loss of generality in its first two rows and first four columns. Then in order to avoid $L_{1}$ and $L_{3}$, the first two rows have zeros elsewhere. Hence after permuting rows, we can decompose $C_{r}$ as

$$
\left.C_{r}=\left[\begin{array}{ccc|c}
1 & 1 & 0 & 00 \cdots 00 \\
1 & 0 & 1 & 00 \cdots 00 \\
\hline 1 & 1 & 1 & H_{111} \\
0 & 0 & 0 & H_{000} \\
0 & 1 & 1 & H_{011} \\
1 & 0 & 1 & H_{101} \\
1 & 1 & 0 & H_{110} \\
0 & 0 & 1 & H_{001} \\
0 & 1 & 0 & H_{010} \\
1 & 0 & 0 & H_{100}
\end{array}\right]\right\} m-3
$$

Here $H_{\alpha}$ is the matrix with $\left|C_{r}\right|-3$ columns, of all rows which appear under the two rows of zeros in the first row, and to the right of copies of the $1 \times 3$ binary triplet $\alpha$ in the first three columns.

In order to avoid $L_{2}$ with row 1 of $C_{r}, H_{111}$ has no configuration $\left[\begin{array}{ll}1 & 0\end{array}\right]$. Hence each row of $H_{111}$ is either all zeros or all ones. To avoid $L_{1}$ with row $1, H_{000}$ has only zeros. To avoid $L_{1}$ with row 1 , both $H_{011}$ and $H_{101}$ avoid [ 110$]$. Similarly with row $2, H_{110}$ avoids $\left[\begin{array}{ll}1 & 0\end{array}\right]$. Hence each row of $H_{011}, H_{101}$ and $H_{110}$ is either all zeros or all ones. To avoid $L_{1}$ with row $1, H_{001}$ has only ones, and similarly with row $2, H_{010}$ has only ones.

Thus each row above $H_{100}$ is either all zeros or all ones, and so $H_{100}$ is simple. In order to avoid $L_{1}$ with row 1 of $C_{r}, H_{100}$ has no configuration $\left[\begin{array}{ll}1 & 1\end{array}\right]$. Let $H_{100}$ have $n$ rows, where $n \leqslant m-3$. Then by Theorem 1.8 with $p=1$ and $q=0,\left|H_{100}\right| \leqslant \operatorname{forb}\left(n,\left[\begin{array}{ll}1 & 1\end{array}\right]\right)=$ $n+1 \leqslant m-2$. But $\left|H_{100}\right|=\left|C_{r}\right|-3 \geqslant m-2$, and so we conclude that $n=m-3$ and that $H_{100} \in \operatorname{ext}\left(m-2,\left[\begin{array}{ll}1 & 1\end{array}\right]\right)$. It follows from Theorem 1.8 that, after permuting columns, $H_{100}=\left[I_{m-3} \mathbf{0}_{m-3}\right]$. Hence

$$
C_{r}=\left[\begin{array}{ccc|cccc}
1 & 1 & 0 & 0 & 0 \cdots 0 & 0 \\
1 & 0 & 1 & 0 & 0 \cdots 0 & 0 \\
\hline 1 & 0 & 0 & 1 & 0 \cdots 0 & 0 \\
1 & 0 & 0 & 0 & 1 \cdots 0 & 0 \\
\vdots & \vdots & \vdots & \vdots & \vdots & \vdots & \vdots \\
1 & 0 & 0 & 0 & 0 & \cdots & 0
\end{array}\right]=\left[\mathbf{1}_{m-1} I_{m-1} \mathbf{0}_{m-1}\right]
$$

and so $C_{r} \in \operatorname{ext}\left(m-1, \mathbf{1}_{2} \mathbf{0}_{1}\right)$, as desired.

Noting that $F_{2}^{c}, F_{3}, F_{4}, F_{5}$ are configurations in $F_{8}$, we have obtained the bounds in Table 2. It is not clear that this result has the best choices $F_{6}, F_{7}, F_{8}$. We now use 
Lemma 3.1 and Theorem 3.2 to establish results for a family of forbidden configurations which contain $2 \cdot \mathbf{1}_{k-1} \mathbf{0}_{1}$ and whose bounds and extremal matrices are given by those for $2 \cdot \mathbf{1}_{k-1} \mathbf{0}_{1}$. For an $m_{1} \times n_{1}$ matrix $A$ and an $m_{2} \times n_{2}$ matrix $B$ we use the notation $A \times B$ or

$$
\begin{gathered}
A \\
\times \\
B
\end{gathered}
$$

to denote the $\left(m_{1}+m_{2}\right) \times n_{1} n_{2}$ matrix corresponding to the cartesian product of the columns so that the columns of $A \times B$ are formed by placing a column of $A$ on top of a column of $B$ in all possible ways. An example is that $K_{4}=K_{1} \times K_{3}$. We note that if $A, B$ are both simple then $A \times B$ is simple. Also note that $A \times B$ need not be $B \times A$ as matrices although they would be the same if one performed row permutations.

Theorem 3.3 Let $F$ be either

$$
\left[\begin{array}{llll}
1 & 1 & 0 & 0 \\
1 & 0 & 1 & 0 \\
0 & 1 & 0 & 0
\end{array}\right] \quad \text { or }\left[\begin{array}{llll}
1 & 1 & 0 & 0 \\
1 & 0 & 1 & 0 \\
0 & 0 & 0 & 0
\end{array}\right] \text { or }\left[\begin{array}{lllll}
1 & 1 & 1 & 0 & 0 \\
1 & 0 & 0 & 0 & 0 \\
0 & 1 & 0 & 1 & 0
\end{array}\right] \text {. }
$$

For $k \geqslant 4$, we let $F(k)$ be the $k$-rowed matrix

$$
F(k)=\left[\mathbf{1}_{k-1} \mathbf{0}_{1} \mid K_{k-3} \times F\right]=k-1\left\{\left[\begin{array}{cc}
1 & \\
\vdots & K_{k-3} \\
1 & \times \\
1 & F \\
0 &
\end{array}\right] .\right.
$$

Let $F(3)$ be any one of $F_{6}, F_{7}, F_{8}$ from Theorem 3.2. Suppose that $m \geqslant 3$. Then for all $k$ such that $3 \leqslant k \leqslant m$,

$$
\operatorname{ext}(m, F(k))=\operatorname{ext}\left(m, 2 \cdot \mathbf{1}_{k-1} \mathbf{0}_{1}\right)=\operatorname{ext}\left(m, \mathbf{1}_{k} \mathbf{0}_{1}\right) .
$$

Proof: We induct on $m$ using Lemma 3.1, where our base case $m=3$ is clear. We suppose that the result holds for $m-1$, for all $k$. Now let $\ell$ be any integer such that $3 \leqslant \ell \leqslant m$. Note that $F(\ell)$ is not simple, so any $\ell$-rowed simple matrix avoids $F(\ell)$. Hence the result follows if $\ell=m$. On the other hand, if $\ell=3$, then the result follows from Theorem 3.2. Thus we assume $4 \leqslant \ell \leqslant m-1$.

Let $A \in \operatorname{ext}(m, F(\ell))$. Now $F(\ell)$ is of the form given in the statement of Lemma 3.1, so it will suffice to show that the two hypotheses of the Lemma hold. The hypothesis (i) follows from our induction hypothesis. Now suppose that $r \in[m]$ such that, in the row- $r$ decomposition (2) of $A,\left|C_{r}\right|>\operatorname{forb}\left(m-1, \mathbf{1}_{\ell-1}\right)$. Then since forb $\left(m-1, \mathbf{1}_{\ell-1} \mathbf{0}_{1}\right)=$ $1+$ forb $\left(m-1, \mathbf{1}_{\ell-1}\right)$ for $\ell \leqslant m-1$, by our induction hypothesis we have $\left|C_{r}\right| \geqslant$ forb $(m-$ $\left.1, \mathbf{1}_{\ell-1} \mathbf{0}_{1}\right)=$ forb $(m-1, F(\ell-1))$. But since $A$ avoids $F(\ell)$, following Lemma 2.1 we see that $C_{r}$ avoids $F(\ell-1)$. For this we need $\ell \geqslant 4$. Hence $C_{r} \in \operatorname{ext}(m-1, F(\ell-1))=$ 
$\operatorname{ext}\left(m-1, \mathbf{1}_{\ell-1} \mathbf{0}_{1}\right)$, again by our induction hypothesis. This provides hypothesis (ii) of Lemma 3.1.

The notation $F(k)$ and $F(3)$ matches at $k=3$ if we define $K_{0} \times F$ appropriately. Note that $F(k)$ has only one repeated column. An example with more repeated columns is in Theorem 4.1.

The following self-complementary cases $F_{9}$ and $F_{10}$ have both $2 \cdot \mathbf{1}_{2} \mathbf{0}_{1}$ and $2 \cdot \mathbf{1}_{1} \mathbf{0}_{2}$ as the critical substructures. Note that our result below gives an example of a $4 \times 4$ configuration $F_{10}$ which has a $3 \times 2$ critical substructure.

$$
F_{9}=\left[\begin{array}{llll}
1 & 1 & 1 & 1 \\
1 & 1 & 0 & 0 \\
0 & 0 & 0 & 0
\end{array}\right]=K_{1} \times\left(2 \cdot \mathbf{1}_{1} \mathbf{0}_{1}\right), \quad F_{10}=\left[\begin{array}{llll}
1 & 1 & 1 & 1 \\
1 & 1 & 0 & 0 \\
1 & 0 & 1 & 0 \\
0 & 0 & 0 & 0
\end{array}\right]
$$

Theorem 3.4 For $m \geqslant 3$ we have

$$
\operatorname{forb}\left(m, F_{9}\right)=\operatorname{forb}\left(m, F_{10}\right)=\operatorname{forb}\left(m, 2 \cdot \mathbf{1}_{2} \mathbf{0}_{1}\right)=\operatorname{forb}\left(m, 2 \cdot \mathbf{1}_{1} \mathbf{0}_{2}\right)=\left(\begin{array}{c}
m \\
2
\end{array}\right)+m+2 .
$$

Proof: We induct on $m$, where our base case $m=3$ is clear. We suppose that the result is true for $m-1$, where $m \geqslant 4$, and let $A \in \operatorname{ext}\left(m, F_{10}\right)$. Since $|A| \geqslant \operatorname{forb}\left(m, 2 \cdot \mathbf{1}_{2} \mathbf{0}_{1}\right)=$ $\left(\begin{array}{c}m \\ 2\end{array}\right)+m+2$, it will suffice to show that $|A| \leqslant\left(\begin{array}{c}m \\ 2\end{array}\right)+m+2$. If $A$ has both $I_{m}$ and $I_{m}^{c}$, then $A$ avoids $F_{1,1,1,1}$ of $F_{10}$, whence by Theorem $1.9|A| \leqslant \operatorname{forb}\left(m, F_{1,1,1,1}\right)=4 m-4 \leqslant$ $\left(\begin{array}{c}m \\ 2\end{array}\right)+m+2$. Otherwise, in the row- $r$ decomposition (2) of $A$ for some $r \in[m], C_{r}$ is missing either the column of 1's or the column of 0's. Stripping off the second row from $F_{10}$, we see that $C_{r}$ has no $F_{1,1,0,1}$. Since the column of 1's and the column of 0's do not contribute to $F_{1,1,0,1}$, we have using Theorem $1.8\left|C_{r}\right| \leqslant$ forb $\left(m-1, F_{1,1,0,1}\right)-1=m$. Since $\left|\left[B_{r} C_{r} D_{r}\right]\right| \leqslant \operatorname{forb}\left(m-1, F_{10}\right)=\left(\begin{array}{c}m-1 \\ 2\end{array}\right)+(m-1)+2$, the result follows.

It may be verified that $\operatorname{ext}\left(m, F_{9}\right)=\operatorname{ext}\left(m, 2 \cdot \mathbf{1}_{2} \mathbf{0}_{1}\right) \cup \operatorname{ext}\left(m, 2 \cdot \mathbf{1}_{1} \mathbf{0}_{2}\right)$. For $F_{10}$ one might imagine that $\operatorname{ext}\left(m, F_{10}\right)=\operatorname{ext}\left(m, 2 \cdot \mathbf{1}_{2} \mathbf{0}_{1}\right) \cup \operatorname{ext}\left(m, 2 \cdot \mathbf{1}_{1} \mathbf{0}_{2}\right)$. While this is probably true for $m \geqslant 5$, the argument would be delicate since, for $m=4$, an extremal construction which departs from this pattern is the $4 \times 12$ matrix which has exactly two columns with 2 ones, which are complementary, and all other columns on 4 rows.

\section{Critical substructure $\mathbf{1}_{k}$}

Let

$$
F_{11}=\left[\begin{array}{lll}
1 & 1 & 1 \\
1 & 1 & 1 \\
1 & 0 & 0 \\
1 & 0 & 0
\end{array}\right]
$$

The following result considers a $k$-rowed $F$ with $2^{k-4}$ pairs of repeated columns. 
Theorem 4.1 Assume $m \geqslant 5$. Then forb $\left(m, F_{11}\right)=\operatorname{forb}\left(m, \mathbf{1}_{4}\right)$. Assume $k \geqslant 5$ and $m \geqslant k+1$. Then

$$
\operatorname{forb}\left(m, K_{k-4} \times F_{11}\right)=\operatorname{forb}\left(m, \mathbf{1}_{k}\right)=\sum_{i=0}^{k-1}\left(\begin{array}{c}
m \\
i
\end{array}\right)
$$

Proof: We begin by establishing forb $\left(m, F_{11}\right)=$ forb $\left(m, \mathbf{1}_{4}\right)=\left(\begin{array}{c}m \\ 3\end{array}\right)+\left(\begin{array}{c}m \\ 2\end{array}\right)+\left(\begin{array}{c}m \\ 1\end{array}\right)+\left(\begin{array}{c}m \\ 0\end{array}\right)$. Let $A \in \operatorname{ext}\left(m, F_{11}\right)$. Apply standard induction as in (1) obtaining $B, C, D$. It suffices to show $|C| \leqslant\left(\begin{array}{c}m-1 \\ 2\end{array}\right)+\left(\begin{array}{c}m-1 \\ 1\end{array}\right)+\left(\begin{array}{c}m-1 \\ 0\end{array}\right)$. We deduce that $C$ has no configuration

$$
F_{2}=\left[\begin{array}{lll}
1 & 1 & 1 \\
1 & 0 & 0 \\
1 & 0 & 0
\end{array}\right]
$$

and so by Theorem $3.2\left(F_{2}^{c}\right.$ is in $\left.F_{8}\right),|C| \leqslant\left(\begin{array}{c}m-1 \\ 2\end{array}\right)+\left(\begin{array}{c}m-1 \\ 1\end{array}\right)+\left(\begin{array}{c}m-1 \\ 0\end{array}\right)+1$ with equality if and only if $C \in \operatorname{ext}\left(m-1,2 \cdot \mathbf{1}_{1} \mathbf{0}_{2}\right)$. In that case $C$ has a column of 1 's and hence $A$ has a column of 1 's and hence $A$ has no configuration $2 \cdot \mathbf{1}_{2} \mathbf{0}_{2}$. But then by Theorem 1.8 ,

$$
|A| \leqslant \operatorname{forb}\left(m, 2 \cdot \mathbf{1}_{2} \mathbf{0}_{2}\right)=\left(\begin{array}{c}
m \\
2
\end{array}\right)+2 m+2 \leqslant\left(\begin{array}{c}
m \\
3
\end{array}\right)+\left(\begin{array}{c}
m \\
2
\end{array}\right)+\left(\begin{array}{c}
m \\
1
\end{array}\right)+\left(\begin{array}{c}
m \\
0
\end{array}\right)
$$

for $m \geqslant 5$. Thus $|C| \leqslant\left(\begin{array}{c}m-1 \\ 2\end{array}\right)+\left(\begin{array}{c}m-1 \\ 1\end{array}\right)+\left(\begin{array}{c}m-1 \\ 0\end{array}\right)$ and we have established forb $\left(m, F_{11}\right)=$ forb $\left(m, \mathbf{1}_{4}\right)$.

We now apply Remark 2.2 with $p=k$ and $q=0$ to obtain the bound for any $F=$ $K_{k-4} \times F_{11}$ by induction on $k$ for $k \geqslant 5$.

\section{$5 \quad$ Critical substructures $\mathbf{1}_{k-1} \mathbf{0}_{1}$ and $k \times 2 F$}

In this and the next two sections we consider forb $\left(m, F_{a, b, c, d}\right)$ for cases with $c, d \in\{0,1\}$. We can restrict our attention to cases with $a \geqslant d$ and $b \geqslant c$. Note that forb $\left(m, F_{a, b, 0,0}\right)=$ forb $\left(m, \mathbf{1}_{a+b}\right)$ follows from Theorem 1.2 and Theorem 1.8 handles forb $\left(m, F_{a, 0,0, d}\right)=$ forb $\left(m, F_{a, 1,0, d}\right)$. For some cases given below, the column $\mathbf{1}_{a+b} \mathbf{0}_{c+d}$ is a critical substructure of $F_{a, b, c, d}$ (when $a=d$ one also considers $\mathbf{1}_{a+c} \mathbf{0}_{b+d}$ ).

Problem 5.1 Find some conditions on $a, b, c, d$ so that

$$
\operatorname{forb}\left(m, F_{a, b, c, d}\right)=\operatorname{forb}\left(m, \mathbf{1}_{a+b} \mathbf{0}_{c+d}\right) .
$$

We expect that some appropriate inequalities $a \geqslant d+c_{1}$ and $b \geqslant c+c_{2}$ for constants $c_{1}, c_{2}$ will suffice. Note that $F_{0,2,1,0}$ in [4] and $F_{0,2,2,0}, F_{2,1,1,0}$ in [3] do not follow the pattern and we expect many such instances. We provide some cases where the bounds of Problem 5.1 are true using inductive arguments. We develop an analogue of Lemma 3.1. The following Lemma will only be applied for 2-columned $F$ but we state it in more generality. 
Lemma 5.2 Suppose that $k \geqslant 2$, and $G_{1}$ and $G_{2}$ are $k$-1-rowed simple matrices, possibly empty, whose columns have at least 2 zeros. Let $G=\left[\begin{array}{c|c}11 \cdots 1 & 00 \cdots 0 \\ G_{1} & G_{2}\end{array}\right]$, and let $F$ be the $k$-rowed simple matrix

$$
F=\left[\mathbf{1}_{k-1} \mathbf{0}_{1} \mid G\right]=k-1\left\{\left[\begin{array}{cc}
1 & \\
1 & \\
\vdots & G \\
1 & \\
0 &
\end{array}\right]=k-1\left\{\left[\begin{array}{c|c|c}
1 & 11 \cdots 1 & 00 \cdots 0 \\
1 & & \\
\vdots & G_{1} & G_{2} \\
1 & & \\
0 & &
\end{array}\right] .\right.\right.
$$

Let $A \in \operatorname{ext}(m, F)$, where $m \geqslant k$. Suppose that we know the following three things:

(i) $\operatorname{ext}(m-1, F)=\operatorname{ext}\left(m-1, \mathbf{1}_{k-1} \mathbf{0}_{1}\right)$.

(ii) $\operatorname{forb}(m, G)<\operatorname{forb}\left(m, \mathbf{1}_{k-1} \mathbf{0}_{1}\right)$.

(iii) For each $r \in[m]$ where $C_{r}$ does not have $\mathbf{1}_{m-1}$ in the row-r decomposition (2) of $A$, we have $C_{r} \in \operatorname{ext}\left(m-1, \mathbf{1}_{k-2}\right)$.

Then

$$
\operatorname{ext}(m, F)=\operatorname{ext}\left(m, \mathbf{1}_{k-1} \mathbf{0}_{1}\right) .
$$

Proof: Assume $A \in \operatorname{ext}(m, F)$ and (i), (ii), (iii) hold. Since $F$ has $\mathbf{1}_{k-1} \mathbf{0}_{1}$, we have $|A| \geqslant \operatorname{forb}\left(m, \mathbf{1}_{k-1} \mathbf{0}_{1}\right)$, and it will suffice to show that $A$ avoids $\mathbf{1}_{k-1} \mathbf{0}_{1}$. We first show that $A$ does not have the configuration $I_{m}^{c}=K_{m}^{m-1}$. Otherwise, $A$ has $I_{k}^{c}$ on every $k$-set of rows, and so $A$ has no $G$. Then by our assumption (ii), $|A| \leqslant \operatorname{forb}(m, G)<\operatorname{forb}\left(m, \mathbf{1}_{k-1} \mathbf{0}_{1}\right)$, a contradiction. Hence $A$ does not have $I_{m}^{c}$.

Thus for some $r \in[m]$, in the row- $r$ decomposition (2) of $A$, neither $B_{r}$ nor $C_{r}$ has $\mathbf{1}_{m-1}$. By our assumption (iii), $C_{r} \in \operatorname{ext}\left(m-1, \mathbf{1}_{k-2}\right)$. Hence $\left|\left[B_{r} C_{r} D_{r}\right]\right|=|A|-\left|C_{r}\right| \geqslant$ $\operatorname{forb}\left(m, \mathbf{1}_{k-1} \mathbf{0}_{1}\right)-\operatorname{forb}\left(m-1, \mathbf{1}_{k-2}\right)=\operatorname{forb}\left(m-1, \mathbf{1}_{k-1} \mathbf{0}_{1}\right)=\operatorname{forb}(m-1, F)$ by Remark 2.2 and our assumption (i). Since $\left[B_{r} C_{r} D_{r}\right]$ avoids $F$, we have $\left[B_{r} C_{r} D_{r}\right] \in \operatorname{ext}(m-1, F)=$ $\operatorname{ext}\left(m-1, \mathbf{1}_{k-1} \mathbf{0}_{1}\right)$ by (i). To show that $A$ avoids $\mathbf{1}_{k-1} \mathbf{0}_{1}$, it will suffice to show that $B_{r}$ avoids $\mathbf{1}_{k-1}$ and $C_{r}$ avoids $\mathbf{1}_{k-2}$ and $D_{r}$ avoids $\mathbf{1}_{k-2} \mathbf{0}_{1}$. Since $B_{r}$ avoids $\mathbf{1}_{k-1} \mathbf{0}_{1}$ and does not have $\mathbf{1}_{m-1}$, it also avoids $\mathbf{1}_{k-1}$. We have that $C_{r}$ avoids $\mathbf{1}_{k-2}$. We now show that $D_{r}$ avoids $\mathbf{1}_{k-2} \mathbf{0}_{1}$.

Because $C_{r} \in \operatorname{ext}\left(m-1, \mathbf{1}_{k-2}\right), C_{r}$ has a copy of $\left[K_{k-1}^{0} K_{k-1}^{1} K_{k-1}^{2} \cdots K_{k-1}^{k-3}\right]$ on each $k$ - 1-tuple of its rows. Hence $C_{r}$ has a copy of every row permutation of $G_{1}$ and $G_{2}$ on each $k$-1-tuple of its rows. Now if we look for the top row of $F$ in row $r$ of $A$, we see that $D_{r}$ has no $\mathbf{1}_{k-2} \mathbf{0}_{1}$, as desired.

We now establish sufficient conditions to obtain the hypothesis (ii) above.

Lemma 5.3 For positive integers $m, x$ and $y$, where $m \geqslant x+y+2$, we have

$$
\sum_{i=0}^{x}\left(\begin{array}{c}
m \\
i
\end{array}\right)+\sum_{i=0}^{y}\left(\begin{array}{c}
m \\
i
\end{array}\right)<\sum_{i=0}^{x+y}\left(\begin{array}{c}
m \\
i
\end{array}\right)
$$


Proof: Without loss of generality, suppose $x \leqslant y$. Then it will suffice to show that

$$
\sum_{i=0}^{x}\left(\begin{array}{c}
m \\
i
\end{array}\right)<\sum_{i=y+1}^{x+y}\left(\begin{array}{c}
m \\
i
\end{array}\right) .
$$

If $x=1$, then since $2 \leqslant y+1 \leqslant m-2$, we have

$$
\left(\begin{array}{c}
m \\
y+1
\end{array}\right) \geqslant\left(\begin{array}{c}
m \\
2
\end{array}\right)>\left(\begin{array}{c}
m \\
0
\end{array}\right)+\left(\begin{array}{c}
m \\
1
\end{array}\right)
$$

giving (10). Otherwise, $x \geqslant 2$. Then for $i=1, \ldots, x$, we have $i<x+y+1-i<m-i$, whence $\left(\begin{array}{c}m \\ i\end{array}\right)<\left(\begin{array}{c}m \\ x+y+1-i\end{array}\right)$. Thus

$$
\sum_{i=0}^{x}\left(\begin{array}{c}
m \\
i
\end{array}\right)<\sum_{i=1}^{x}\left(\begin{array}{c}
m \\
x+y+1-i
\end{array}\right)=\sum_{i=y+1}^{x+y}\left(\begin{array}{c}
m \\
i
\end{array}\right)
$$

again giving (10).

We note that $x, y \geqslant 1$ and $m \geqslant x+y+2$ are also necessary conditions for the above inequality to hold, for integers $m, x$ and $y$. Now taking $x=p-1$ and $y=q-1$, we have the following Corollary, which can be more readily applied to hypothesis ii) of Lemma 5.2 .

Corollary 5.4 For $p, q \geqslant 2$ and $m \geqslant p+q$,

$$
\operatorname{forb}\left(m, \mathbf{1}_{p} \mathbf{0}_{q}\right)<\operatorname{forb}\left(m, \mathbf{1}_{p+q-1}\right)<\operatorname{forb}\left(m, \mathbf{1}_{p+q-1} \mathbf{0}_{1}\right) .
$$

For our next main result Theorem 5.6 we establish base cases first. The bound for $F_{1,2,1,0}$ is in [3] but we need $\operatorname{ext}(m, F)$ for this case.

Theorem 5.5 Let $m$ be a positive integer. Then $\operatorname{ext}\left(m, F_{1,2,1,0}\right)=\operatorname{ext}\left(m, \mathbf{1}_{3} \mathbf{0}_{1}\right)$.

Proof: We induct on $m$. The result is clear for $m \leqslant 3$. For $m=4$, let $A \in \operatorname{ext}\left(4, F_{1,2,1,0}\right)$. Then $|A| \geqslant \operatorname{forb}\left(4, \mathbf{1}_{3} \mathbf{0}_{1}\right)=12$. We use the notation $\mathcal{A}$ to denote the set system associated with $A$. Thus $\mathcal{A}$ is missing at most 4 of the 16 subsets of $\{1,2,3,4\}$. Note that if $\mathcal{A}$ has a set with 3 elements (e.g. $\{1,2,3\}$ ), then since $A$ has no $F_{1,2,1,0}$, three sets with 2 elements are missing from $\mathcal{A}$ (e.g. $\{1,4\},\{2,4\},\{3,4\}$ ). Similarly if $A$ has two (or more) sets of three elements then it must be missing at least 5 sets of 2 elements. Since $|A|=12$, it follows that $\mathcal{A}$ has no sets with 3 elements and $\mathcal{A}$ has all other subsets of $\{1,2,3,4\}$, and so $A \in \operatorname{ext}\left(4, \mathbf{1}_{3} \mathbf{0}_{1}\right)$. This establishes our base cases $m \leqslant 4$.

Now suppose that the result holds for $m-1$, where $m \geqslant 5$. Let $A$ be any matrix in $\operatorname{ext}(m, F)$. Then by Corollary 6.2 (from the next section), it will suffice to show that $C \in$ $\operatorname{ext}\left(m-1, \mathbf{1}_{2}\right)$ in the standard decomposition (1) of $A$. We have $|A| \geqslant \operatorname{forb}\left(m, \mathbf{1}_{3} \mathbf{0}_{1}\right)$ and by induction $|[B C D]| \leqslant \operatorname{forb}\left(m-1, \mathbf{1}_{3} \mathbf{0}_{1}\right)$, whence $|C|=|A|-|[B C D]| \geqslant \operatorname{forb}\left(m, \mathbf{1}_{3} \mathbf{0}_{1}\right)-$ forb $\left(m-1, \mathbf{1}_{3} \mathbf{0}_{1}\right)=\operatorname{forb}\left(m-1, \mathbf{1}_{2}\right)=m($ Remark 2.2). Let $\gamma$ be a column in $C$ with 
greatest column sum $s \geqslant 1$ (or set of largest cardinality in $\mathcal{C}$ ). If $s=1$ then $C \in$ $\operatorname{ext}\left(m-1, \mathbf{1}_{2}\right)$, so we proceed by contradiction and suppose that $s \geqslant 2$.

Define the notation that for a column $\alpha$ we let $S(\alpha)$ denote the subset in $\mathcal{A}$ corresponding to the column $\alpha$. We say that two columns $\alpha$ and $\beta$ are incomparable if $S(\alpha) \nsubseteq S(\beta)$ and $S(\beta) \nsubseteq S(\alpha)$. Note that $C$ has no configurations $F_{0,2,1,0}, F_{1,1,1,0}$ or $F_{1,2,0,0}$ Hence if $\alpha$ and $\beta$ are incomparable columns in $C$, then $\alpha$ and $\beta$ give the configuration $I_{2}=F_{0,1,1,0}$ and so by the forbidden configurations $F_{0,2,1,0}, F_{1,1,1,0}$ for $C$ we deduce $\alpha$ and $\beta$ each have exactly 1 one. That is, if $X, Y \in \mathcal{C}$ such that $|X| \neq 1$ or $|Y| \neq 1$, then $X \subseteq Y$ or $Y \subseteq X$.

In particular, for each $t \geqslant 2, \mathcal{C}$ has at most one set with $t$ elements. Also, since $\gamma$ has maximum sum, $Z \subseteq S(\gamma)$ for all $Z \in \mathcal{C}$. Given the forbidden configuration $F_{1,2,0,0}$, $|Z| \geqslant s-1$ for all non-empty $Z \in \mathcal{C}$. It follows that if $s \geqslant 3$, then $\mathcal{C}$ has at most 3 sets (at most one each of size $s$ and $s-1$, and possibly the empty set). But $|\mathcal{C}| \geqslant m \geqslant 5$, so $s=2$. Then because $Z \subseteq S(\gamma)$ for all $Z \in \mathcal{C}$, we have $|\mathcal{C}| \leqslant 4$ (since $S(\gamma)$ has 4 subsets). This contradiction completes the proof.

For the following theorem note that we are computing forb $\left(m, F_{b, a, 1,0}\right)$ using $a, b$ in an unusual order.

Theorem 5.6 For $m \geqslant 1, a \geqslant 2$ and $b \geqslant 1$,

$$
\operatorname{ext}\left(m, F_{a, b, 0,1}\right)=\operatorname{ext}\left(m, F_{b, a, 1,0}\right)=\operatorname{ext}\left(m, \mathbf{1}_{a+b} \mathbf{0}_{1}\right) .
$$

Proof: We induct on $m$ using Lemma 5.2, where our base cases $m \leqslant 3$ are clear. We suppose that the result holds for $m-1$, for all $a \geqslant 2$ and $b \geqslant 1$. Now let $a$ and $b$ be any integers such that $a \geqslant 2$ and $b \geqslant 1$. If $m \leqslant a+b$, then the result is clear, while if $a=2$ and $b=1$, then we may use Theorem 1.8 for $F_{2,1,0,1}$ and Theorem 5.5 for $F_{1,2,1,0}$. Thus we assume that $m \geqslant a+b+1$, and either $a \geqslant 3$ or $b \geqslant 2$.

Let $A \in \operatorname{ext}\left(m, F_{a, b, 0,1}\right) \cup \operatorname{ext}\left(m, F_{b, a, 1,0}\right)$. Note that $F_{a, b, 0,1}$ and $F_{b, a, 1,0}$ are of the form given in the statement of Lemma 5.2 with $k=a+b$, so it will suffice to show that the three hypotheses of the Lemma hold. The hypothesis (i) follows from our induction hypothesis. The hypothesis (ii) follows from Corollary 5.4, since the second columns of $F_{a, b, 0,1}$ and $F_{b, a, 1,0}$ both have at least 2 ones and at least 2 zeros.

Now we show that hypothesis (iii) holds. Suppose that for some $r \in[m]$ such that $\left|C_{r}\right|$ does not have $\mathbf{1}_{m-1}$ in the row- $r$ decomposition (2) of $A$. We have that $|A| \geqslant$ forb $\left(m, \mathbf{1}_{a+b} \mathbf{0}_{1}\right)$, since $\mathbf{1}_{a+b} \mathbf{0}_{1}$ is in both $F_{a, b, 0,1}$ and $F_{b, a, 1,0}$. By induction $\left|B_{r} C_{r} D_{r}\right| \leqslant$ forb $\left(m-1, \mathbf{1}_{a+b} \mathbf{0}_{1}\right)$. Thus using Remark 2.2, we have $\left|C_{r}\right|=|A|-\left|\left[B_{r} C_{r} D_{r}\right]\right| \geqslant$ forb $(m-$ $\left.1, \mathbf{1}_{a+b-1}\right)=$ forb $\left(m-1, \mathbf{1}_{a+b-1} \mathbf{0}_{1}\right)-1$. Now if $a \geqslant 3$, then $C_{r}$ either does not have $F_{a-1, b, 0,1}$ or $F_{b, a-1,1,0}$, while if $b \geqslant 2$, then $C_{r}$ either does not have $F_{a, b-1,0,1}$ or $F_{b-1, a, 1,0}$. By our induction hypothesis, the $m-1$-rowed extremal matrices for each of these four Forbidden Configurations are the same as those for $\mathbf{1}_{a+b-1} \mathbf{0}_{1}$. Since $\mathbf{1}_{m-1}$ does not contribute to any of these four Forbidden Configurations, we have $\left[C_{r} \mathbf{1}_{m-1}\right] \in \operatorname{ext}\left(m-1, \mathbf{1}_{a+b-1} \mathbf{0}_{1}\right)$, whence $C_{r} \in \operatorname{ext}\left(m-1, \mathbf{1}_{a+b-1}\right)$. 


\section{Some self-complementary $k \times 2 F$}

Above we established the extremal matrices for $F_{a, b, 0,1}$ and $F_{b, a, 1,0}$, where $a \geqslant 2$ and $b \geqslant 1$. We now examine some self-complementary cases $F_{1, b, 0,1}$. We use Corollary 6.3 with the result for $F_{1,2,0,1}$ as a base case. We prove that the extremal matrices either avoid the first column or the second column of the Forbidden Configuration and hence establish that $\mathbf{1}_{1+b} \mathbf{0}$ and $\mathbf{1}_{1} \mathbf{0}_{b+1}$ are critical substructures for $F_{1, b, 0,1}$.

The corollaries of the following lemma will be used in our proofs for 2-columned configurations. They allow us to assert in an induction argument that, if we know that $C_{r}$ has a certain structure, then $A$ also does.

Lemma 6.1 Suppose that $a, b, c, d$ are non-negative integers such that $a \geqslant d, b>c$, and $b \geqslant 2$. Let $A \in \operatorname{ext}\left(m, F_{a, b, c, d}\right)$, where $c+d \geqslant 1$ and $m \geqslant a+b+c+d$. Suppose for some $r \in[m]$ that in the row-r decomposition (2) of $A$ we have $C_{r} \in \operatorname{ext}\left(m-1, \mathbf{1}_{a+b-1} \mathbf{0}_{c+d-1}\right)$. Then $A \in \operatorname{ext}\left(m, \mathbf{1}_{a+b} \mathbf{0}_{c+d}\right)$.

Proof: Assume $A \in \operatorname{ext}\left(m, F_{a, b, c, d}\right)$. Since $\mathbf{1}_{a+b} \mathbf{0}_{c+d}$ is the first column of $F_{a, b, c, d}$, we have $|A|=\operatorname{forb}\left(m, F_{a, b, c, d}\right) \geqslant \operatorname{forb}\left(m, \mathbf{1}_{a+b} \mathbf{0}_{c+d}\right)$. It will suffice to show that $A$ avoids $\mathbf{1}_{a+b} \mathbf{0}_{c+d}$. Because $C_{r}$ avoids $\mathbf{1}_{a+b-1} \mathbf{0}_{c+d-1}$, it will in turn suffice to show that $D_{r}$ avoids $\mathbf{1}_{a+b-1} \mathbf{0}_{c+d}$ and $B_{r}$ avoids $\mathbf{1}_{a+b} \mathbf{0}_{c+d-1}$.

Since $b>c, C_{r}$ has all columns with $a+c-1$ ones on $m-1$ rows. Then $D_{r}$ avoids $\mathbf{1}_{a+b-1} \mathbf{0}_{c+d}$, since otherwise a column $\gamma$ in $D_{r}$ with $\mathbf{1}_{a+b-1} \mathbf{0}_{c+d}$ can be matched with a suitable column $\beta$ of $C_{r}$ with $a+c-1$ ones to give $F_{a, b, c, d}$ in $A$ using the 1's in row $r$. Similarly, if $c \geqslant 1$, then $B_{r}$ avoids $\mathbf{1}_{a+b} \mathbf{0}_{c+d-1}$, since otherwise a column $\gamma$ in $B_{r}$ with $\mathbf{1}_{a+b} \mathbf{0}_{c+d-1}$ can be paired with a suitable column $\beta$ of $C_{r}$ with $a+c-1$ ones to give $F_{a, b, c, d}$ in $A$ where $\gamma$ has a 0 in row $r$ but we choose $\beta$ with a 1 in row $r$. If $c=0$ then since $b \geqslant 2$, $C_{r}$ has all columns with $a$ ones on $m-1$ rows. Hence $B_{r}$ again avoids $\mathbf{1}_{a+b} \mathbf{0}_{d-1}$, since otherwise a column $\gamma$ in $B_{r}$ with $\mathbf{1}_{a+b} \mathbf{0}_{\max \{0, d-1\}}$ can be matched with a suitable column $\beta$ of $C_{r}$ with $a$ ones to give $F_{a, b, c, d}$ in $A$ using 0 's in row $r$.

We state two corollaries to this Lemma. If a $(0,1)$-matrix $F$ is a row and column permutation of its complement $F^{c}$, then we say that $F$ is self-complementary. We will use the first corollary below when $F$ is not self-complementary $(a>d)$, and the second when $F$ is self-complementary $(a=d)$.

Corollary 6.2 Suppose that $a, b, c, d$ are non-negative integers such that $a>d, b>c$, and $b \geqslant 2$, and let $m \geqslant a+b+c+d$. Suppose that for every matrix $A \in \operatorname{ext}\left(m, F_{a, b, c, d}\right)$, there exists an $r \in[m]$, depending on $A$, such that $C_{r} \in \operatorname{ext}\left(m-1, \mathbf{1}_{a+b-1} \mathbf{0}_{c+d-1}\right)$ in the row-r decomposition (2) of $A$. Then

$$
\operatorname{ext}\left(m, F_{a, b, c, d}\right)=\operatorname{ext}\left(m, \mathbf{1}_{a+b} \mathbf{0}_{c+d}\right) .
$$

Proof: We get $\operatorname{ext}\left(m, F_{a, b, c, d}\right) \subseteq \operatorname{ext}\left(m, \mathbf{1}_{a+b} \mathbf{0}_{c+d}\right)$ by Lemma 6.1, whence the result follows since $\mathbf{1}_{a+b} \mathbf{0}_{c+d}$ is a configuration of $F_{a, b, c, d}$. 
Corollary 6.3 Suppose that $a, b, c$ are non-negative integers such that $b>c$ and $b \geqslant 2$, and let $m \geqslant 2 a+b+c$. Suppose that for every matrix $A \in \operatorname{ext}\left(m, F_{a, b, c, a}\right)$, there exists an $r \in[m]$, depending on $A$, such that $C_{r} \in \operatorname{ext}\left(m-1, \mathbf{1}_{a+b-1} \mathbf{0}_{a+c-1}\right) \cup \operatorname{ext}\left(m-1, \mathbf{1}_{a+c-1} \mathbf{0}_{a+b-1}\right)$ in the row-r decomposition (2) of $A$. Then

$$
\operatorname{ext}\left(m, F_{a, b, c, d}\right)=\operatorname{ext}\left(m, \mathbf{1}_{a+b} \mathbf{0}_{a+c}\right) \cup \operatorname{ext}\left(m, \mathbf{1}_{a+c} \mathbf{0}_{a+b}\right) .
$$

Proof: For any $A \in \operatorname{ext}\left(m, F_{a, b, c, a}\right)$, we take $r$ such that $C_{r} \in \operatorname{ext}\left(m-1, \mathbf{1}_{a+b-1} \mathbf{0}_{a+c-1}\right) \cup$ $\operatorname{ext}\left(m-1, \mathbf{1}_{a+c-1} \mathbf{0}_{a+b-1}\right)$. Then either $C_{r}$ or $C_{r}^{c}$ is in $\operatorname{ext}\left(m-1, \mathbf{1}_{a+b-1} \mathbf{0}_{a+c-1}\right)$, and since $F_{a, b, c, a}$ is self-complementary, we have $A^{c} \in \operatorname{ext}\left(m, F_{a, b, c, a}\right)$. Hence by Lemma 6.1 with $d=$ $a$, either $A$ or $A^{c}$ is in $\operatorname{ext}\left(m, \mathbf{1}_{a+b} \mathbf{0}_{a+c}\right)$, whence $A \in \operatorname{ext}\left(m, \mathbf{1}_{a+b} \mathbf{0}_{a+c}\right) \cup \operatorname{ext}\left(m, \mathbf{1}_{a+c} \mathbf{0}_{a+b}\right)$. The result follows since $\mathbf{1}_{a+b} \mathbf{0}_{a+c}$ and $\mathbf{1}_{a+c} \mathbf{0}_{a+b}$ are both configurations of $F_{a, b, c, a}$.

The bound $\operatorname{forb}\left(m, F_{1201}\right)$ is given in [6] but we also need ext $\left(m, F_{1201}\right)$.

Theorem 6.4 Let $m$ be a positive integer. Then

$$
\operatorname{ext}\left(m, F_{1,2,0,1}\right)=\operatorname{ext}\left(m, \mathbf{1}_{3} \mathbf{0}_{1}\right) \cup \operatorname{ext}\left(m, \mathbf{1}_{1} \mathbf{0}_{3}\right) .
$$

Proof: We induct on $m$. Our base cases $m \leqslant 3$ are clear. Now suppose that the result holds for $m-1$, where $m \geqslant 4$. Let $A$ be any matrix in $\operatorname{ext}\left(m, F_{1,2,0,1}\right)$. Then $A$ does not have both $I_{m}$ and $I_{m}^{c}$, since otherwise it has $F$. Hence for some $r \in[m]$, in the row- $r$ decomposition (2) of $A, C_{r}$ is missing either $\mathbf{1}_{m-1}$ or $\mathbf{0}_{m-1}$. By Corollary 6.3, it will suffice to show that $C_{r} \in \operatorname{ext}\left(m-1, \mathbf{1}_{2}\right) \cup \operatorname{ext}\left(m-1, \mathbf{0}_{2}\right)$.

Now to avoid $F_{1,2,0,1}$ in $A$, we deduce that $C_{r}$ has no configuration $F_{0,2,0,1}, F_{1,1,0,1}$ or $F_{1,2,0,0}$. If two columns of $C_{r}$ contain $F_{1,0,0,1}$, then the two columns must also contain $F_{1,1,0,1}\left(C_{r}\right.$ is simple $)$, a contradiction. So $C_{r}$ has no $F_{1,0,0,1}$. Now given $|A| \geqslant \operatorname{forb}\left(m, \mathbf{1}_{3} \mathbf{0}_{1}\right)$ and by induction $\left|B_{r} C_{r} D_{r}\right| \leqslant$ forb $\left(m-1, \mathbf{1}_{3} \mathbf{0}_{1}\right)$ and we may deduce by Remark 2.2 that $\left|C_{r}\right|=|A|-\left|\left[B_{r} C_{r} D_{r}\right]\right| \geqslant \operatorname{forb}\left(m-1, \mathbf{1}_{2}\right)=m=$ forb $\left(m-1, F_{1,0,0,1}\right)-1$. Thus $C_{r}$ has either $\mathbf{0}_{m-1}$ or $\mathbf{1}_{m-1}$ since both columns do not contribute to $F_{1,0,0,1}$. Suppose that $C_{r}$ has $\mathbf{0}_{m-1}$. Then because $C_{r}$ avoids $F_{0,2,0,1}$, it avoids $\mathbf{1}_{2} \mathbf{0}_{1}$. Then since $C_{r}$ is missing $\mathbf{1}_{m-1}$, it also avoids $\mathbf{1}_{2}$, whence $C_{r} \in \operatorname{ext}\left(m-1, \mathbf{1}_{2}\right)$. Similarly, if $C_{r}$ has $\mathbf{1}_{m-1}$, then $C_{r} \in \operatorname{ext}\left(m-1, \mathbf{0}_{2}\right)$.

Theorem 6.5 For $m \geqslant 1$ and $b \geqslant 2$,

$$
\operatorname{ext}\left(m, F_{1, b, 0,1}\right)=\operatorname{ext}\left(m, \mathbf{1}_{b+1} \mathbf{0}_{1}\right) \cup \operatorname{ext}\left(m, \mathbf{1}_{1} \mathbf{0}_{b+1}\right) .
$$

Proof: We induct on $m$, where our base case $m=1$ is clear. We suppose that the result holds for $m-1$, for all $b \geqslant 2$, where $m \geqslant 2$. Let $b$ be any integer such that $b \geqslant 2$. If $m \leqslant b+1$ the result is clear, while if $b=2$ the result is true by Theorem 6.4 . Thus we suppose that $3 \leqslant b \leqslant m-2$. Let $A \in \operatorname{ext}\left(m, F_{1, b, 0,1}\right)$. Then $A$ does not have both $I_{m}$ and $I_{m}^{c}$, since otherwise it has $F_{1, b, 0,1}$. Hence for some $r \in[m], C_{r}$ is missing either $\mathbf{1}_{m-1}$ or $\mathbf{0}_{m-1}$ in the row- $r$ decomposition (2) of $A$.

Now given $|A| \geqslant \operatorname{forb}\left(m, \mathbf{1}_{1+b} \mathbf{0}_{1}\right)$ and, by induction, $\left|B_{r} C_{r} D_{r}\right| \leqslant \operatorname{forb}\left(m, \mathbf{1}_{1+b} \mathbf{0}_{1}\right)$, we have by Remark 2.2 that $|C|=|A|-|[B C D]| \geqslant \operatorname{forb}\left(m-1, \mathbf{1}_{b}\right)=\operatorname{forb}\left(m-1, \mathbf{1}_{b} \mathbf{0}_{1}\right)-1$. 
Now since $C_{r}$ has no $F_{1, b-1,0,1}$, by our induction hypothesis we have $\left|C_{r}\right| \leqslant$ forb $(m-$ $\left.1, F_{1, b-1,0,1}\right)=\operatorname{forb}\left(m-1, \mathbf{1}_{b} \mathbf{0}_{1}\right)$. In particular, $C_{r}$ is missing at most one of $\mathbf{1}_{m-1}, \mathbf{0}_{m-1}$ since neither contribute to $F_{1, b-1,0,1}$. Suppose that $C_{r}$ has $\mathbf{0}_{m-1}$. Then since $C_{r}$ avoids $F_{0, b, 0,1}$, it has no $\mathbf{1}_{b} \mathbf{0}_{1}$. Since, in this case, $C_{r}$ is missing $\mathbf{1}_{m-1}$, we conclude that $C_{r}$ avoids $\mathbf{1}_{b}$. Similarly, if $C_{r}$ has $\mathbf{1}_{m-1}$, then it avoids $\mathbf{0}_{b}$. Thus $C_{r} \in \operatorname{ext}\left(m-1, \mathbf{1}_{b}\right) \cup \operatorname{ext}\left(m-1, \mathbf{0}_{b}\right)$. The result now follows by Corollary 6.3, with $a=1$ and $c=0$.

In a similar way we can prove that for $3 \leqslant b \leqslant m-2, \operatorname{ext}\left(m, F_{0, b, 1,0}\right)=\operatorname{ext}\left(m, \mathbf{1}_{b} \mathbf{0}_{1}\right) \cup$ $\operatorname{ext}\left(m, \mathbf{1}_{1} \mathbf{0}_{b}\right)$, the bound having been established in [3].

\section{Critical substructures $\mathbf{1}_{k-2} \mathbf{0}_{2}$ and $k \times 2 F$}

In this section we compute $\operatorname{forb}\left(m, F_{a, b, 1,1}\right)$ for $a \geqslant 1$ and $b \geqslant 2$, where we separately prove the base case $F_{1,2,1,1}$. We note that, in all of our previous generalizations, we were examining $F$ for which forb $(m, F)=\operatorname{forb}\left(m, \mathbf{1}_{k-1} \mathbf{0}_{1}\right)$. In such cases, we could get an upper bound on $C_{r}$ which differed by only one from its lower bound. However, in this case, our upper and lower bounds on $C_{r}$ differ by $m-1$. Because of this, we apply a more direct argument.

Theorem 7.1 Let $m$ be a positive integer. Then for $m \geqslant 1$,

$$
\operatorname{ext}\left(m, F_{1,2,1,1}\right)=\operatorname{ext}\left(m, \mathbf{1}_{3} \mathbf{0}_{2}\right) \cup \operatorname{ext}\left(m, \mathbf{1}_{2} \mathbf{0}_{3}\right) .
$$

Proof: We induct on $m$. Our base cases $m \leqslant 4$ are clear. Now suppose that the result holds for $m-1$, where $m \geqslant 5$. Let $A$ be any matrix in $\operatorname{ext}\left(m, F_{1,2,1,1}\right)$. Apply the decomposition of (1). Then by Corollary 6.3, it will suffice to show that $C \in \operatorname{ext}(m-$ $\left.1, \mathbf{1}_{2} \mathbf{0}_{1}\right) \cup \operatorname{ext}\left(m-1, \mathbf{1}_{1} \mathbf{0}_{2}\right)$. We have $|A| \geqslant$ forb $\left(m, \mathbf{1}_{3} \mathbf{0}_{2}\right)$ and assert by induction $|[B C D]| \leqslant$ forb $\left(m-1, \mathbf{1}_{3} \mathbf{0}_{2}\right)$, so by Remark $2.2,|C|=|A|-|[B C D]| \geqslant \operatorname{forb}\left(m-1, \mathbf{1}_{2} \mathbf{0}_{1}\right)=m+1$. Let $C^{\prime}$ be the matrix formed by removing $\mathbf{1}_{m-1}$ and $\mathbf{0}_{m-1}$ from $C$, if either is present. Then $\left|C^{\prime}\right| \geqslant m-1$. Let $\gamma$ be a column in $C^{\prime}$ of greatest column sum in $C^{\prime}$ where $\gamma$ has $s$ ones. If $s=1$ then $C \in \operatorname{ext}\left(m-1, \mathbf{1}_{2} \mathbf{0}_{1}\right)$. Hence we assume that $s \geqslant 2$, and show that then $C \in \operatorname{ext}\left(m-1, \mathbf{1}_{1} \mathbf{0}_{2}\right)$. It will suffice to show that $C^{\prime}$ has all columns with exactly 1 zero, and no others.

Note that $C^{\prime}$ has no $F_{1,1,1,1}, F_{1,2,1,0}$, and no $F_{0,2,1,1}$. Hence $C^{\prime}$ has neither

$$
\left[\begin{array}{ll}
0 & \\
1 & 1 \\
1 & 0 \\
0 & 1
\end{array}\right] \text { nor }\left[\begin{array}{ll}
1 & \\
0 & 0 \\
1 & 0 \\
0 & 1
\end{array}\right]
$$

where the blanks can be filled with a 0 or 1 . Hence if $\alpha$ and $\beta$ are two incomparable columns in $C^{\prime}$, then either $\alpha$ and $\beta$ both have exactly 1 one, both have exactly 1 zero, or are complements.

$C^{\prime}$ also has no $F_{1,2,0,1}$. Since columns in $C^{\prime}$ have a one and a zero, we conclude that if $\alpha$ and $\beta$ are columns in $C^{\prime}$ such that $S(\beta) \subset S(\alpha)$, then $\beta$ has exactly one less 1 than $\alpha$. 
Now we show that $s=m-2$. We proceed by contradiction and suppose that $s \leqslant m-3$. If $\gamma^{c}$ is in $C^{\prime}$, then every other column in $C^{\prime}$ is incomparable either with $\gamma$ or with $\gamma^{c}$. Since $\gamma$ and $\gamma^{c}$ both have at least 2 ones and at least 2 zeros, we get $\left|C^{\prime}\right|=2$. Since $\left|C^{\prime}\right| \geqslant m-1 \geqslant 4$, we conclude that $\gamma^{c}$ is not in $C^{\prime}$. Hence, since $\gamma$ has at least 2 ones and at least 2 zeros, no columns in $C^{\prime}$ are incomparable with $\gamma$. Then because $\gamma$ has maximum sum, all sets in $C^{\prime}$ are subsets of $S(\gamma)$. If $s=2$, since $\emptyset \notin C^{\prime}$, we have $\left|C^{\prime}\right| \leqslant 3$, which contradicts $\left|C^{\prime}\right| \geqslant 4$. Hence $s \geqslant 3$, whence all columns in $C^{\prime}$, other than $\gamma$, have $s-1$ ones. Since any two such columns are incomparable and have at least 2 ones and at least 2 zeros, they must be complementary. This gives $\left|C^{\prime}\right| \leqslant 3$, again a contradiction.

Thus $s=m-2$, that is, $\gamma$ has exactly 1 zero. We now show that $\gamma^{c}$ is not in $C^{\prime}$. Otherwise, every column $\alpha$ in $C^{\prime}$, other than $\gamma$ and $\gamma^{c}$, is incomparable with $\gamma$ or $\gamma^{c}$, whence $\alpha$ has exactly 1 one or exactly 1 zero. If $\alpha$ has exactly 1 one, then $S(\alpha) \subset S(\gamma)$, and hence $\alpha$ has exactly $m-3$ ones. If $\alpha$ has exactly 1 zero, then $S\left(\gamma^{c}\right) \subset S(\alpha)$, and hence $\alpha$ has exactly 2 ones. In either case we get a contradiction, so we conclude that $\gamma^{c}$ is not in $C^{\prime}$.

Thus every column $\beta \neq \gamma$ in $C^{\prime}$ either has exactly 1 zero (if $\beta$ and $\gamma$ are incomparable) or exactly 2 zeros (if $S(\beta) \subset S(\gamma)$ ). Suppose $\beta$ has exactly 2 zeros and hence at least 2 ones. Then $\beta$ cannot be incomparable to any other column of $C^{\prime}$. Thus $S(\beta) \subset S(\gamma)$. It follows that $S(\beta)$ is a subset of every other set in $C^{\prime}$ (all columns in $C^{\prime}$ have at most 2 zeros). Since $[m-1] \notin C^{\prime}$, we get $\left|C^{\prime}\right| \leqslant 3$, which contradicts $\left|C^{\prime}\right| \geqslant 4$. Hence $C^{\prime}$ has no columns with exactly 2 zeros, so all columns have exactly 1 zero. Since $\left|C^{\prime}\right| \geqslant m-1, C^{\prime}$ has all such columns, as desired.

Theorem 7.2 For $m \geqslant 1, a \geqslant 1$ and $b \geqslant 2$,

$$
\operatorname{ext}\left(m, F_{a, b, 1,1}\right)=\left\{\begin{array}{ll}
\operatorname{ext}\left(m, \mathbf{1}_{a+b} \mathbf{0}_{2}\right), & \text { if } a \geqslant 2 \\
\operatorname{ext}\left(m, \mathbf{1}_{1+b} \mathbf{0}_{2}\right) \cup \operatorname{ext}\left(m, \mathbf{1}_{2} \mathbf{0}_{1+b}\right), & \text { if } a=1
\end{array} .\right.
$$

Proof: We induct on $m$, where our base cases $m \leqslant 4$ are clear. We suppose that the result is true for $m-1$, for all $a \geqslant 1$ and $b \geqslant 2$, where $m \geqslant 5$. Let $a \geqslant 1$ and $b \geqslant 2$. If $m \leqslant a+b+1$, then the result is clear, while if $a=1$ and $b=2$, the result follows from Theorem 7.1 above. Hence we assume that $4 \leqslant a+b \leqslant m-2$. In particular, $m \geqslant 6$. Let $A \in \operatorname{ext}\left(m, F_{a, b, 1,1}\right)$. By Corollaries 6.2 and 6.3 , it will suffice to show that in the standard decomposition of $A$ in (1) that $C \in \operatorname{ext}\left(m-1, \mathbf{1}_{a+b-1} \mathbf{0}_{1}\right)$ if $a \geqslant 2$, and $C \in \operatorname{ext}\left(m-1, \mathbf{1}_{b} \mathbf{0}_{1}\right) \cup \operatorname{ext}\left(m-1, \mathbf{1}_{1} \mathbf{0}_{b}\right)$ if $a=1$.

We have $|A| \geqslant \operatorname{forb}\left(m, \mathbf{1}_{a+b} \mathbf{0}_{2}\right)$ and by induction $|[B C D]| \leqslant$ forb $\left(m-1, \mathbf{1}_{a+b} \mathbf{0}_{2}\right)$, hence by Remark 2.2, $|C| \geqslant|A|-|[B C D]|=$ forb $\left(m-1, \mathbf{1}_{a+b-1} \mathbf{0}_{1}\right)$. We now consider two cases, $b \geqslant 3$ and $b=2$.

Case $(\mathbf{I}), b \geqslant 3$ : In this case we use that $C$ has no $F_{a, b-1,1,1}$, and so by our induction hypothesis,

$$
|C| \leqslant \operatorname{forb}\left(m-1, F_{a, b-1,1,1}\right)=\text { forb }\left(m-1, \mathbf{1}_{a+b-1} \mathbf{0}_{2}\right)=\text { forb }\left(m-1, \mathbf{1}_{a+b-1} \mathbf{0}_{1}\right)+m-1 .
$$

Hence $C$ is missing at most $m-1$ columns which do not contribute to $F_{a, b-1,1,0}$. We now consider two subcases, $a \geqslant 2$ and $a=1$. 
Subcase (Ii), $a \geqslant 2$ : Suppose that $C$ has a column with exactly 1 zero (say in row $j$ ). Then because $C$ has no $F_{a, b, 0,1}$, it is missing at least $\left(\begin{array}{c}m-2 \\ a\end{array}\right) \geqslant\left(\begin{array}{c}m-2 \\ 2\end{array}\right)>m-1$ columns with exactly $a$ ones (those with a zero in row $j$ ). Since these columns do not contribute to $F_{a, b-1,1,1}$, this is a contradiction. Thus $C$ has no columns from $I_{m-1}^{c}$. Because there are $m-1$ such columns and they do not contribute to $F_{a, b-1,1,1}$, we conclude that $\left[C I_{m-1}^{c}\right] \in \operatorname{ext}\left(m-1, F_{a, b-1,1,1}\right)$. Since $\operatorname{ext}\left(m-1, F_{a, b-1,1,1}\right)=\operatorname{ext}\left(m-1, \mathbf{1}_{a+b-1} \mathbf{0}_{2}\right)$ by our induction hypothesis, $C \in \operatorname{ext}\left(m-1, \mathbf{1}_{a+b-1} \mathbf{0}_{1}\right)$, as desired.

Subcase (Iii), $a=1$ : As in the case above, if $C$ has a column from $I_{m-1}^{c}$, then it is missing at least $\left(\begin{array}{c}m-2 \\ a\end{array}\right)=m-2$ columns from $I_{m-1}$. Similarly, since $F_{1, b, 0,1}$ is selfcomplementary, if $C$ has a column from $I_{m-1}$, then it is missing at least $m-2$ columns from $I_{m-1}^{c}$. Note that columns in $I_{m-1}^{c}$ and $I_{m-1}$ do not contribute to $F_{1, b-1,1,1}$. Hence $C$ is missing either $I_{m-1}^{c}$ or $I_{m-1}$, since otherwise it is missing at least $2(m-2)>m-1$ columns which do not contribute to $F_{1, b-1,1,1}$. Suppose that $C$ is missing $I_{m-1}^{c}$. Then $C$ has $I_{m-1}$ and, because $C$ avoids $F_{0, b, 1,1}$, it avoids the first column $\mathbf{1}_{b} \mathbf{0}_{2}$ of $F_{0, b, 1,1}$. Since $C$ is missing $I_{m-1}^{c}, C$ avoids $\mathbf{1}_{b} \mathbf{0}_{1}$, whence $C \in \operatorname{ext}\left(m-1, \mathbf{1}_{b} \mathbf{0}_{1}\right)$. Similarly, if $C$ is missing $I_{m-1}$, then $C \in \operatorname{ext}\left(m-1, \mathbf{1}_{1} \mathbf{0}_{b}\right)$. Thus $C \in \operatorname{ext}\left(m-1, \mathbf{1}_{b} \mathbf{0}_{1}\right) \cup \operatorname{ext}\left(m-1, \mathbf{1}_{1} \mathbf{0}_{b}\right)$, as desired. Case (II), $b=2$ : In this case we use that $C$ has no $F_{a-1, b, 1,1}$ using $a \geqslant 2$, and so by our induction hypothesis,

$$
|C| \leqslant \operatorname{forb}\left(m-1, F_{a-1, b, 1,1}\right)=\operatorname{forb}\left(m-1, \mathbf{1}_{a+b-1} \mathbf{0}_{2}\right)=\text { forb }\left(m-1, \mathbf{1}_{a+b-1} \mathbf{0}_{1}\right)+m-1 .
$$

Since columns from $I_{m-1}^{c}$ do not contribute to $F_{a-1, b, 1,1}$, as in Subcase (Ii) above, it will suffice to show that $C$ is missing $I_{m-1}^{c}$. We proceed by contradiction and suppose that $C$ has at least one column from $I_{m-1}^{c}$. We consider two subcases - when $C$ has exactly one such column, and when $C$ has at least 2 such columns.

Subcase (IIi), $C$ has exactly one column from $I_{m-1}^{c}$ : In this case, after permuting rows and columns, we can decompose $C$ as

$$
C=\left[\begin{array}{c|c|c}
1 & & \\
\vdots & X_{1} & X_{2} \\
1 & & \\
1 & & \\
0 & 00 \cdots 0 & 11 \cdots 1
\end{array}\right] .
$$

Since $C$ avoids $F_{a, 2,0,1}$ and $F_{a, 2,1,0}$, both $\left[X_{1} \mathbf{1}_{m-2}\right]$ and $X_{2}$ avoid $\mathbf{1}_{a} \mathbf{0}_{2}$. Also, since $C$ has only one column from $I_{m-1}^{c}, X_{2}$ is missing the $m-2$ columns in $I_{m-2}^{c}$. Since these columns do not contribute to $\mathbf{1}_{a} \mathbf{0}_{2}$, we have

$$
\begin{aligned}
|C| & =\left|\left[X_{1} \mathbf{1}_{m-2}\right]\right|+\left|X_{2}\right| \\
& \leqslant 2 \cdot \operatorname{forb}\left(m-2, \mathbf{1}_{a} \mathbf{0}_{2}\right)-(m-2) \\
& =\text { forb }\left(m-2, \mathbf{1}_{a} \mathbf{0}_{1}\right)+\text { forb }\left(m-2, \mathbf{1}_{a-1}\right)+\left(\begin{array}{c}
m-2 \\
a-1
\end{array}\right)+2 m-3-m+2 \\
& =\text { forb }\left(m-1, \mathbf{1}_{a} \mathbf{0}_{1}\right)+\left(\begin{array}{c}
m-2 \\
a-1
\end{array}\right)+m-1,
\end{aligned}
$$

where the last line uses Remark 2.2. Now we asserted

$$
|C| \geqslant \operatorname{forb}\left(m-1, \mathbf{1}_{a+1} \mathbf{0}_{1}\right)=\operatorname{forb}\left(m-1, \mathbf{1}_{a} \mathbf{0}_{1}\right)+\left(\begin{array}{c}
m-1 \\
a
\end{array}\right),
$$


whence $\left(\begin{array}{c}m-2 \\ a-1\end{array}\right)+m-1 \geqslant\left(\begin{array}{c}m-1 \\ a\end{array}\right)$. Using Pascal's Identity we obtain $m-1 \geqslant\left(\begin{array}{c}m-2 \\ a\end{array}\right)$. This is a contradiction, since $m \geqslant 6$.

Subcase (IIii), $C$ has at least 2 columns from $I_{m-1}^{c}$ : Let two such columns be $\alpha$ and $\beta$. Then after permuting rows and columns, we can decompose $C$ as

$$
C=\left[\begin{array}{cc|c|c|c|c}
1 & 1 & & & & \\
\vdots & \vdots & Y_{1} & Y_{2} & Y_{3} & Y_{4} \\
1 & 1 & & & & \\
1 & 0 & 00 \cdots 0 & 11 \cdots 1 & 11 \cdots 1 & 00 \cdots 0 \\
0 & 1 & 00 \cdots 0 & 11 \cdots 1 & 00 \cdots 0 & 11 \cdots 1
\end{array}\right] .
$$

Since $C$ avoids $F_{a, 2,0,1}$, looking at $\alpha$ we see that $Y_{1}$ avoids $\mathbf{1}_{a} \mathbf{0}_{1}$. Since $C$ avoids $F_{a, 2,1,0}$, looking at $\alpha$ we see that $Y_{2}$ avoids $\mathbf{1}_{a-1} \mathbf{0}_{2}$.

Since $C$ avoids $F_{a, 2,0,1}$, looking at $\alpha$ we see that $\left[Y_{3} \mathbf{1}_{m-3}\right]$ avoids $\mathbf{1}_{a-1} \mathbf{0}_{2} . C$ also avoids $F_{a, 2,1,0}$, so looking at $\beta$ we see that $\left[Y_{3} \mathbf{1}_{m-3}\right]$ also avoids $\mathbf{1}_{a} \mathbf{0}_{1}$. Hence $\left[Y_{3} \mathbf{1}_{m-3}\right]$ avoids $\mathbf{1}_{a-1} \mathbf{0}_{1}$. Similarly, $\left[Y_{4} \mathbf{1}_{m-3}\right]$ avoids $\mathbf{1}_{a-1} \mathbf{0}_{1}$. Thus applying Remark 2.2,

$$
\begin{aligned}
|C| & =\left|Y_{1}\right|+\left|Y_{2}\right|+\left|\left[Y_{3} \mathbf{1}_{m-3}\right]\right|+\left|\left[Y_{4} \mathbf{1}_{m-3}\right]\right| \\
& \leqslant \operatorname{forb}\left(m-3, \mathbf{1}_{a} \mathbf{0}_{1}\right)+\operatorname{forb}\left(m-3, \mathbf{1}_{a-1} \mathbf{0}_{2}\right)+2 \cdot \operatorname{forb}\left(m-3, \mathbf{1}_{a-1} \mathbf{0}_{1}\right) \\
& =2 \cdot\left(\operatorname{forb}\left(m-3, \mathbf{1}_{a} \mathbf{0}_{2}\right)+\operatorname{forb}\left(m-3, \mathbf{1}_{a-1} \mathbf{0}_{1}\right)\right)-(m-3)-\left(\begin{array}{c}
m-3 \\
a-1
\end{array}\right) \\
& \leqslant 2 \cdot\left(\operatorname{forb}\left(m-3, \mathbf{1}_{a} \mathbf{0}_{2}\right)+\operatorname{forb}\left(m-3, \mathbf{1}_{a-1} \mathbf{0}_{1}\right)\right)-(m-2) \\
& =2 \cdot \operatorname{forb}\left(m-2, \mathbf{1}_{a} \mathbf{0}_{2}\right)-(m-2),
\end{aligned}
$$

which is a contradiction as in Subcase IIi.

These results conclude our proof of the various cases in Theorem 1.10.

\section{Critical substructure $3 \cdot \mathbf{1}_{k-1}$}

We now handle the final $3 \times 3$ matrix. Let

$$
\left.F_{1}(k)=\left[\begin{array}{ccc}
1 & 1 & 1 \\
1 & 1 & 1 \\
\vdots & \vdots & \vdots \\
1 & 1 & 1 \\
1 & 0 & 0
\end{array}\right]\right\} k-1
$$

Note that $F_{1}(3)$ is $F_{1}$ in Table 2 . The generality of this result for larger $k$ costs nothing.

Theorem 8.1 Let $m, k$ be given.

$$
\operatorname{forb}\left(m, F_{1}(k)\right)=\operatorname{forb}\left(m, 3 \cdot \mathbf{1}_{k-1}\right) \leqslant \frac{k+1}{k}\left(\begin{array}{c}
m \\
k-1
\end{array}\right)+\left(\begin{array}{c}
m \\
k-2
\end{array}\right)+\cdots+\left(\begin{array}{c}
m \\
0
\end{array}\right) .
$$

with equality if there exists a design on $[\mathrm{m}]$ of blocks of size $k$ such that for each subset $S \in\left(\begin{array}{c}{[m]} \\ k-1\end{array}\right)$, there is exactly one block of size $k$ containing it. 
Proof: Let $k \geqslant 3$ be given. Note that $3 \cdot \mathbf{1}_{k-1}$ is a configuration of $F_{1}(k)$. We use Theorem 1.6 for forb $\left(m, 3 \cdot \mathbf{1}_{k-1}\right)$. Much of the argument is given in terms of sets.

For $k=3$, we can construct matrices achieving the bound as follows. For $m \equiv$ $1,3(\bmod 6)$, there is a Steiner triple system on $m$ points yielding an $m \times \frac{1}{3}\left(\begin{array}{c}m \\ 2\end{array}\right)$ simple matrix $M_{m}$ of columns of column sum 3 and containing no configuration $\left[\begin{array}{l}1 \\ 11\end{array}\right]$. Thus $\left[K_{m}^{0} K_{m}^{1} K_{m}^{2} M_{m}\right]$ avoids $F_{1}(3)$ and achieves the upper bound. Similarly if there exists a $(k-1)$-design on $[m]$ of blocks of size $k$ such that for each subset of $[m]$ of size $k-1$, there is exactly one block of size $k$ containing it, let $M_{m}$ be the associated $m \times \frac{1}{k}\left(\begin{array}{c}m \\ k-1\end{array}\right)$ incidence matrix. Thus $\left[K_{m}^{0} K_{m}^{1} \cdots K_{m}^{k-1} M_{m}\right]$ avoids $F_{1}(k)$ and achieves the upper bound. The existence of the block design would require $m, k$ satisfying some easy congruences and having $m$ large enough.

To prove the bound, let $m, k$ be given. Let $A$ be an $m$-rowed simple matrix with no configuration $F_{1}(k)$. We may assume that $A$ has no column of column sum $m$ since then $A$ has no configuration $2 \cdot \mathbf{1}_{k-1} \mathbf{0}_{1}$ and we have by Theorem 1.8 forb $\left(m, 2 \cdot \mathbf{1}_{k-1} \mathbf{0}_{1}\right)=$ $\left(\begin{array}{c}m \\ k-1\end{array}\right)+\cdots+\left(\begin{array}{c}m \\ 1\end{array}\right)+\left(\begin{array}{c}m \\ 0\end{array}\right)+\left(\begin{array}{c}m \\ m\end{array}\right)$, which is less than the desired bound for $F_{1}(k)$. We will also assume $A$ has no column of column sum in $\{0,1, \ldots, k-2\}$ and then add $\left(\begin{array}{c}m \\ k-2\end{array}\right)+\cdots+\left(\begin{array}{c}m \\ 1\end{array}\right)+\left(\begin{array}{c}m \\ 0\end{array}\right)$ to the bound for $|A|$ we obtain since if $A$ has no $F_{1}(k)$, then neither does $\left[A K_{m}^{k-2} K_{m}^{k-3} \cdots K_{m}^{0}\right]$.

Let $\mathcal{A} \subseteq 2^{[\mathrm{m}]}$ be the set system associated with $A$. Thus each $B \in \mathcal{A}$ satisfies $k-1 \leqslant$ $|B| \leqslant m-1$. Here we are using $|B|$ to denote the cardinality of the set $B$. Let $S$ be any $(k-1)$-set in $\left(\begin{array}{c}{[m]} \\ k-1\end{array}\right)$ and consider any three sets $B, C, D \in \mathcal{A}$ with $S \subseteq B, C, D$. Then to avoid the configuration $F_{1}(k)$, we must have $B \backslash C, C \backslash B \subseteq D$. Also note that there cannot be a tower of 3 sets $B \subseteq C \subseteq D$ with $B, C, D \in \mathcal{A}$ and $|B| \geqslant k-1$.

We first separate $\mathcal{A}$ into the maximal sets $\mathcal{M}$ in $\mathcal{A}$ of size at least $k$ and the remaining sets $\mathcal{R}$. Thus $\mathcal{R}$ contains any maximal sets of size $k-1$ if any are in $\mathcal{A}$ as well perhaps as other sets. We will then partition $\mathcal{M}=\mathcal{M}^{\prime} \cup \mathcal{M}^{\prime \prime}$ such that each pair of sets in $\mathcal{M}^{\prime}$ intersect in at most $k-2$ elements and we have a matching of the sets $D \in \mathcal{R} \cup \mathcal{M}^{\prime \prime}$ into the sets $S \in\left(\begin{array}{c}{[m]} \\ k-1\end{array}\right)$ (so that if $D$ is matched to $S$ then $S \subseteq D$ ). We have $\left|\mathcal{M}^{\prime}\right| \leqslant \frac{1}{k}\left(\begin{array}{c}m \\ k-1\end{array}\right)$ using a standard pigeonhole argument on the number of columns of column sum at least $k$ with no configuration $2 \cdot \mathbf{1}_{k-1}$ and by the matching we have $\left|\mathcal{R} \cup \mathcal{M}^{\prime \prime}\right| \leqslant\left(\begin{array}{c}m \\ k-1\end{array}\right)$. The bound follows using $|A|=\left|\mathcal{M}^{\prime}\right|+\left|\mathcal{M}^{\prime \prime}\right|+|\mathcal{R}|$.

Assume $T \in \mathcal{A}$ with $|T| \geqslant k$. We deduce that $A$ has no configuration $2 \cdot \mathbf{1}_{k-1} \mathbf{0}_{1}$ on the rows of $T$. We cannot have two distinct sets $C, D \in \mathcal{A}$ with $C \cap T=D \cap T$ and $k-1 \leqslant|C \cap T|,|D \cap T|<|T|$ else we get $F_{1}(k)$ in the columns of $A$ associated with the sets $C, D, T$. Thus we may conveniently define a set of subsets of $[\mathrm{m}]$ :

$$
\mathcal{A}(T)=\{C \cap T: C \in \mathcal{A} \text { and } k-1 \leqslant|C \cap T|<|T|\},
$$

where we note that for each set $E \in \mathcal{A}(T)$, there is a unique set $C \in \mathcal{A}$ with $E=C \cap T$.

For each maximal set $B \in \mathcal{M}$ we show below how to obtain a matching of sets of $\mathcal{A}(B)$ into $\left(\begin{array}{c}B \\ k-1\end{array}\right)$ where a set $D \in \mathcal{A}(B)$ is matched into a set $S \in\left(\begin{array}{c}B \\ k-1\end{array}\right)$ with $S \subseteq D$. At the end we show how to use these matchings, one for each maximal set, to obtain the desired partition $\mathcal{M}=\mathcal{M}^{\prime} \cup \mathcal{M}^{\prime \prime}$ and matching of the sets $\mathcal{M}^{\prime \prime} \cup \mathcal{R}$ into $\left(\begin{array}{c}{[m]} \\ k-1\end{array}\right)$. 
Consider a maximal set $B \in \mathcal{M}$ and the poset on $\mathcal{A}(B)$ ordered by set containment. Extend this to a linear order on all the sets in $\mathcal{A}(B)$ respecting the containment order. Match the sets in turn following this linear order in increasing fashion. For each set $D \in \mathcal{A}(B)$ match $D$ into an unmatched set $S \in\left(\begin{array}{c}B \\ k-1\end{array}\right)$ with $S \subseteq D$. How can this matching process fail? Assume we have reached a set $C$ in our ordering of $\mathcal{A}(B)$ so that some $\left(\begin{array}{c}|C| \\ k-1\end{array}\right)$ sets in $\mathcal{A}(B)$ have been already matched to all $(k-1)$-sets $\left(\begin{array}{c}C \\ k-1\end{array}\right)$. Thus $k \leqslant|C|<|B|$. Now consider the submatrix $A_{C}$ of $A$ consisting of the rows of $C$ and all columns with at least $k-11$ 's and at most $|C|-1$ 1's on the rows of $C$ i.e. the simple matrix associated with $\mathcal{A}(C)$. For $D \in \mathcal{A}(C)$ we have by our above observations that there is a unique set $D^{\prime}$ in $\mathcal{A}$ with $C \cap D^{\prime}=D$ and $B \backslash C \subseteq D^{\prime}$ (if $B \backslash C \nsubseteq D^{\prime}$ then $F_{1}(k)$ is formed by the sets $\left.B, C, D\right)$. Thus there is no configuration $2 \cdot \mathbf{1}_{k-2} \mathbf{0}_{1}$ in $A_{C}$ else, using an element of $B \backslash C$ and set $C$, we will have $F_{1}(k)$. To avoid creating $2 \cdot \mathbf{1}_{k-2} \mathbf{0}_{1}$, we can have at most $(k-1)\left(\begin{array}{c}|C| \\ k-1\end{array}\right)=|C|\left(\begin{array}{c}|C|-1 \\ k-2\end{array}\right)$ configurations $\mathbf{1}_{k-2} \mathbf{0}_{1}$ (where the factor $(k-1)$ accounts for row orderings of $\left.\mathbf{1}_{k-2} \mathbf{0}_{1}\right)$. Each column of $p$ 1's in $A_{C}$ contains at least $\left(\begin{array}{c}p \\ k-2\end{array}\right)(|C|-p)$ configurations $\mathbf{1}_{k-2} \mathbf{0}_{1}$ contained in the rows of $C$, and hence at least $(k-1)(|C|-(k-1))$ such configurations (corresponding to taking $p=k-1$ ). Thus there are at most $\left(\begin{array}{l}|C| \\ k-1\end{array}\right) /(|C|-(k-1))$ columns in $A_{C}$. Hence for $|C|>k$, we have $\left(\begin{array}{c}|C| \\ k-1\end{array}\right)>\left(\begin{array}{l}|C| \\ k-1\end{array}\right) /(|C|-(k-1))$ and so not all sets of $\left(\begin{array}{c}C \\ k-1\end{array}\right)$ can have been already matched when it is time to consider $C$, a contradiction. For $|C|=k$, it is possible that all $\left(\begin{array}{c}|C| \\ k-1\end{array}\right)=k$ subsets have been matched but this results in a special structure. Assume $C=\{1,2, \ldots, k\}$ and $B \backslash C=E$. Then the sets that have been matched to the $k(k-1)$ sets $\left(\begin{array}{c}C \\ k-1\end{array}\right)$ must occur before $C$ in the ordering and so must be the sets $D_{1}=(C \backslash 1) \cup E$, $D_{2}=(C \backslash 2) \cup E, \ldots, D_{k}=(C \backslash k) \cup E$. But given that there are no towers of three sets in $\mathcal{A}$, we deduce that $\mathcal{A}(B)=\left\{D_{1}, D_{2}, \ldots, D_{k}, C\right\}$ and so we choose some $x \in E$ and match $D_{1}$ to the $(k-1)$-set $C \backslash\{1,2\} \cup\{x\}$, match $C$ to the $k-1$-set $C \backslash 1$ and for $j=2,3, \ldots, k$, match $D_{j}$ to the $(k-1)$-set $C \backslash j$. Thus we are able to obtain the desired matching for $\mathcal{A}(B)$.

We now obtain the partition $\mathcal{M}=\mathcal{M}^{\prime} \cup \mathcal{M}^{\prime \prime}$ and matching from $\mathcal{M}^{\prime \prime}$ into $\left(\begin{array}{l}{[m]} \\ k-1\end{array}\right)$. We use the preliminary matchings obtained above. Arbitrarily order the sets in $\mathcal{M}$ as $B_{1}, B_{2}, B_{3}, \ldots$ For each set $C \in \mathcal{A}$, choose the smallest index $i$ so that $\left|C \cap B_{i}\right| \geqslant k-1$. If no such $i$, then $|C|=k-1$ and $C$ is maximal in $\mathcal{A}$. In this case $C \in \mathcal{R}$ and we match $C$ to the set $C$. If $i$ exists and $C=B_{i}$, then $C$ will not get matched and $C$ is put in $\mathcal{M}^{\prime}$. If $C \neq B_{i}$, then $C$ is matched to the same set in $\left(\begin{array}{c}B_{i} \\ k-1\end{array}\right)$ as $C$ is matched to when considering $B_{i}$. If in addition $C \in \mathcal{M}$ then $C$ is put in $\mathcal{M}^{\prime \prime}$. Thus every set $C$ in $\mathcal{R}$ will get matched to some $(k-1)-$ set $S$ in $\left(\begin{array}{l}{[m]} \\ k-1\end{array}\right)$ with $S \subseteq C$.

We first show that we have the desired matching of $\mathcal{M}^{\prime \prime}$ into $\left(\begin{array}{l}{[m]} \\ k-1\end{array}\right)$. Assume the contrary that there are a pair of sets $C, D \in \mathcal{R} \cup \mathcal{M}^{\prime \prime}$ with $|C \cap D| \geqslant k-1$ and assume both are matched to the same set $S \in\left(\begin{array}{l}{[m]} \\ k-1\end{array}\right)$. Then $S \subseteq C \cap D$. Let $i$ be the smallest index of a maximal set $B_{i}$ which intersects $C$ in at least $k-1$ elements and let $j$ be the smallest index of a maximal set $B_{j}$ which intersects $D$ in at least $k-1$ elements. Given that $S \subseteq B_{i}, B_{j}$, we must have $i=j$ and $C \neq B_{i}$ and $D \neq B_{i}$. But when considering the matching for $\mathcal{A}\left(B_{i}\right)$, the two sets $C, D$ will be matched to different sets, a contradiction. 
We now show that $\mathcal{M}^{\prime}$ has no two sets whose intersection is of size $k-1$ or larger. Given any pair of maximal sets $B_{p}, B_{q} \in \mathcal{M}$ with $\left|B_{p} \cap B_{q}\right| \geqslant k-1$ with $p<q$, we can show that $B_{q} \in \mathcal{M}^{\prime \prime}$. Assume $i$ is the smallest index of a maximal set $B_{i}$ which intersects $B_{q}$ in at least $k-1$ elements. Then $i \leqslant p$ and so $B_{i} \neq B_{q}$ and so $B_{q}$ gets matched to some set and $B_{q}$ is put in $\mathcal{M}^{\prime \prime}$.

We have establishes the desired partition and as noted above, this yields our bound. Note that if we have equality in our bound, then $\left|\mathcal{M}^{\prime}\right|=\frac{1}{k}\left(\begin{array}{c}m \\ k-1\end{array}\right)$ and hence the columns will form a $(k-1)$-design on $[m]$ with blocks of size $k$ and $\lambda=1$.

\section{A Difficult Example}

It was noted in [2] that $\frac{29}{21}\left(\begin{array}{c}m \\ 2\end{array}\right)+m+1 \leqslant \operatorname{forb}\left(m, F_{2,1,1,0}\right) \leqslant 2\left(\begin{array}{c}m \\ 2\end{array}\right)+m+1$ and a construction there can be generalized to yield the following result which shows that forb $\left(m, F_{2,1,1,0}\right)$ cannot be a specific quadratic: the coefficient of $\left(\begin{array}{c}m \\ 2\end{array}\right)$ grows with $m$.

Theorem 9.1 Let $c$ be a positive real number. Let $A$ be an $m \times\left(c\left(\begin{array}{c}m \\ 2\end{array}\right)+m+2\right)$ simple matrix with no $F_{2,1,1,0}$. Then for some $M>m$, there is an $M \times\left(\left(c+\frac{2}{m(m-1)}\right)\left(\begin{array}{c}M \\ 2\end{array}\right)+M+2\right)$ simple matrix with no $F_{2,1,1,0}$.

Proof: By results of Wilson [13], for some $M>m$, there is a block design with blocks $\mathcal{B} \subseteq\left(\begin{array}{c}{[M]} \\ m\end{array}\right)$ such that each pair in $\left(\begin{array}{c}{[M]} \\ 2\end{array}\right)$ is contained in a unique block. Thus there are $\frac{2}{m(m-1)}\left(\begin{array}{c}M \\ 2\end{array}\right)$ blocks. We can form an $M \times \frac{2}{m(m-1)}\left(\begin{array}{c}M \\ 2\end{array}\right)$ simple matrix $A^{\prime}$ of columns of sum $m$ corresponding to the blocks. We also create an $M \times\left(c\left(\begin{array}{c}M \\ 2\end{array}\right)\right)$ simple matrix $A^{\prime \prime}$ as follows. Let $\bar{A}$ be the $m \times\left(c \cdot\left(\begin{array}{c}m \\ 2\end{array}\right)\right)$ matrix obtained from $A$ above by deleting the $m+2$ columns of sum $0,1, m$. For each block $B$ (of size $m$ ) of the $\operatorname{design} \mathcal{B}$, we place into $A^{\prime \prime}$ a copy of $\bar{A}$ in the associated rows with 0's in the remaining rows. We note that $\frac{2}{m(m-1)}\left(\begin{array}{c}M \\ 2\end{array}\right) \cdot c\left(\begin{array}{c}m \\ 2\end{array}\right)=c\left(\begin{array}{c}M \\ 2\end{array}\right)$. Thus $A^{\prime \prime}$ is an $M \times\left(c\left(\begin{array}{c}M \\ 2\end{array}\right)\right)$ simple matrix. Now concatenate $A^{\prime}, A^{\prime \prime}$ and the $M+2$ columns of sum $0,1, M$ to form an $M \times\left(c+\frac{2}{m(m-1)}\right)\left(\begin{array}{c}M \\ 2\end{array}\right)+M+2$ simple matrix $A^{\prime \prime \prime}$. If we are looking for $F_{2,1,1,0}$ in $A^{\prime \prime \prime}$, we may ignore the columns of sum 0,1 and $M$. Then a copy of $F_{2,1,1,0}$ must come from a column of sum $m$ and a column from a copy of $A^{\prime \prime}$ on those $m$ rows (in order to find $2 \cdot \mathbf{1}_{2}$ ) but then we cannot find $F_{2,1,1,0}$ in such a pair of columns.

The results extend to $F_{a, 1,1,0}$ for $a>2$. There are other cases where exact bounds would probably be difficult such as $F_{0, b, b-1,0}$ and $F_{1, b, b, 1}$.

\section{Open problems}

Theorem 4.1 considers some $F$ with many repeated pairs of columns. But more should be possible. Perhaps some result such as the following works. 
Problem 10.1 Suppose that $G$ is a k-rowed $(0,1)$-matrix, $k \geqslant 1$, such that forb $(m, G) \leqslant$ forb $\left(m, K_{k-1}\right)$ for all $m \geqslant k+1$. Then for $p \geqslant 1$ and $m \geqslant p+k+1$, is

$$
\operatorname{forb}\left(m, K_{p} \times G\right) \leqslant \operatorname{forb}\left(m, K_{p+k-1}\right) ?
$$

At the very least, we would like the result for $G=\mathbf{1}_{2} \mathbf{0}_{2}$. We were not able to obtain this, and pose it as a problem.

Our results give only partial information for the following.

Problem 10.2 For what 4-rowed $G$ is forb $\left(m, \mathbf{1}_{3} \mathbf{0}_{1}\right)=\operatorname{forb}\left(m,\left[\mathbf{1}_{3} \mathbf{0}_{1} G\right]\right)$ ?

For what 4-rowed $G$ is $\operatorname{forb}\left(m, \mathbf{1}_{4}\right)=\operatorname{forb}\left(m,\left[\mathbf{1}_{4} G\right]\right)$ ?

Problem 10.3 Obtain complete versions of Table 1 and Table 2 for $3 \times 4$ matrices.

It would suffice to obtain exact bounds for the following 12 matrices.

$$
\begin{gathered}
{\left[\begin{array}{llll}
1 & 1 & 1 & 1 \\
1 & 1 & 1 & 1 \\
1 & 0 & 0 & 0
\end{array}\right]\left[\begin{array}{llll}
1 & 1 & 1 & 1 \\
1 & 1 & 1 & 0 \\
1 & 0 & 0 & 1
\end{array}\right]\left[\begin{array}{llll}
1 & 1 & 1 & 1 \\
1 & 1 & 1 & 0 \\
1 & 0 & 0 & 0
\end{array}\right]\left[\begin{array}{llll}
1 & 1 & 1 & 0 \\
1 & 1 & 1 & 0 \\
1 & 0 & 0 & 1
\end{array}\right]\left[\begin{array}{llll}
1 & 1 & 1 & 0 \\
1 & 1 & 1 & 0 \\
1 & 0 & 0 & 0
\end{array}\right]} \\
{\left[\begin{array}{llll}
1 & 1 & 1 & 0 \\
1 & 1 & 1 & 0 \\
0 & 0 & 0 & 0
\end{array}\right]\left[\begin{array}{llll}
1 & 1 & 1 & 1 \\
1 & 1 & 1 & 0 \\
0 & 0 & 0 & 0
\end{array}\right]\left[\begin{array}{llll}
1 & 1 & 1 & 0 \\
1 & 1 & 1 & 0 \\
0 & 0 & 0 & 1
\end{array}\right]\left[\begin{array}{llll}
1 & 1 & 1 & 1 \\
1 & 1 & 1 & 0 \\
0 & 0 & 0 & 1
\end{array}\right]} \\
{\left[\begin{array}{llll}
1 & 1 & 1 & 0 \\
1 & 1 & 0 & 1 \\
0 & 0 & 1 & 1
\end{array}\right]\left[\begin{array}{llll}
1 & 1 & 0 & 0 \\
1 & 1 & 0 & 0 \\
0 & 0 & 1 & 1
\end{array}\right]\left[\begin{array}{llll}
1 & 1 & 1 & 1 \\
1 & 1 & 0 & 0 \\
0 & 0 & 1 & 1
\end{array}\right]}
\end{gathered}
$$

\section{References}

[1] R. P. Anstee, A Survey of Forbidden Configurations results, http://www.math.ubc.ca/ anstee.

[2] R. P. Anstee, F. Barekat, Design Theory and Some Non-simple Forbidden Configurations, submitted to J. Combinatorial Designs.

[3] R. P. Anstee, F. Barekat, A. Sali, Small Forbidden Configurations V: Exact bounds for $4 \times 2$ cases, Studia. Sci. Math. Hun., to appear.

[4] R. P. Anstee, J. R. Griggs, A. Sali, Small Forbidden Configurations, Graphs and Combinatorics 13(1997),97-118.

[5] R. P. Anstee, Z. Füredi, Forbidden Submatrices, Discrete Math. 62(1986),225-243.

[6] R.P. Anstee, R. Ferguson, A. Sali, Small Forbidden Configurations II, Electronic J. Combin. 8(2001), R4 (25pp)

[7] R. P. Anstee, P. Keevash, Pairwise Intersections and Forbidden Configurations, Eur. J. Combin.27(2006), 1235-1248. 
[8] H. O.F. Gronau, An extremal set problem, Studia Sci.Math. Hungar. 15(1980), 2930.

[9] H. J. Ryser, A fundamental matrix equation for finite sets, Proc. Amer. Math. Soc. 34(1972), 332-336.

[10] N. Sauer, On the density of families of sets, J. Combin. Th. Ser A 13(1972), 145-147.

[11] S. Shelah, A combinatorial problem: Stability and order for models and theories in infinitary languages, Pac. J. Math. 41(1972), 247-261.

[12] V. N. Vapnik and A.Ya. Chervonenkis, On the uniform convergence of relative frequencies of events to their probabilities, Th. Prob. and Applics. 16(1971), 264-280.

[13] R. M. Wilson, An existence theory for pairwise balanced designs. III. Proof of the existence conjectures. J. Combinatorial Theory Ser. A 18 (1975), 71-79. 